\title{
Monotonic Algorithms for Transmission Tomography
}

\author{
Hakan Erdoğan, Member, IEEE, and Jeffrey A. Fessler,* Member, IEEE
}

\begin{abstract}
We present a framework for designing fast and monotonic algorithms for transmission tomography penalizedlikelihood image reconstruction. The new algorithms are based on paraboloidal surrogate functions for the log likelihood. Due to the form of the log-likelihood function it is possible to find low curvature surrogate functions that guarantee monotonicity. Unlike previous methods, the proposed surrogate functions lead to monotonic algorithms even for the nonconvex log likelihood that arises due to background events, such as scatter and random coincidences. The gradient and the curvature of the likelihood terms are evaluated only once per iteration. Since the problem is simplified at each iteration, the CPU time is less than that of current algorithms which directly minimize the objective, yet the convergence rate is comparable. The simplicity, monotonicity, and speed of the new algorithms are quite attractive. The convergence rates of the algorithms are demonstrated using real and simulated PET transmission scans.
\end{abstract}

Index Terms - Image reconstruction, maximum likelihood, PET, SPECT.

\section{INTRODUCTION}

A TTENUATION correction is required for quantitatively accurate image reconstruction in emission tomography. The accuracy of this correction is very important in both PET and SPECT [1]. Transmission scans are performed to measure the attenuation characteristics of the object and to determine attenuation correction factors (ACF's) for emission image reconstruction. Conventional smoothing methods for ACF computation are simple and fast, but suboptimal [2], [3]. For low-count transmission scans, statistical reconstruction methods provide lower noise ACF's. However, a drawback of statistical methods is the slow convergence (or possible divergence) of current reconstruction algorithms. This paper describes fast and monotonic algorithms for penalized-likelihood reconstruction of attenuation maps from transmission scan data. These reconstructed attenuation maps can be reprojected to calculate lower noise ACF's for improved emission image reconstruction.

Statistical methods for reconstructing attenuation maps from transmission scans are becoming increasingly important in thorax and whole-body PET imaging, where lower counts and short scan times are typical. 3-D PET systems also

Manuscript received May 21, 1998; revised July 5, 1999. This work was supported in part by the NIH under Grants CA-60711 and CA-54362. The Associate Editor responsible for coordinating the review of this paper and recommending its publication was $\mathrm{C}$. Thompson. Asterisk indicates corresponding author.

H. Erdoğan is with IBM T.J. Watson Research Labs, Yorktown Heights, NY 10598 USA (e-mail: erdogan@us.ibm.com).

*J. A. Fessler is with the Department of Electrical Engineering and Computer Science, University of Michigan, Ann Arbor, MI 48109-2122 USA (e-mail: erdogan@umich.edu; fessler@umich.edu).

Publisher Item Identifier S 0278-0062(99)09052-7. require attenuation correction, which can be done by reprojecting 2-D attenuation maps. SPECT systems with transmission sources are becoming increasingly available where statistical algorithms can be efficiently used for attenuation map reconstructions. For low-count transmission scans, the nonstatistical FBP reconstruction method systematically overestimates attenuation map coefficients, whereas data-weighted least squares methods (WLS) for transmission reconstruction are systematically negatively biased [4]. By accurate statistical modeling, penalized-likelihood reconstruction of attenuation maps eliminates the systematic bias and yields lower variance relative to linear methods. Hence, we focus on penalizedlikelihood image reconstruction rather than WLS in this paper.

There are many reconstruction algorithms based on the Poisson model for transmission measurements. The expectation maximization (EM) algorithm [5], which led to a simple M-step for the emission problem, does not yield a closedform expression for the M-step in the transmission case [6]. Modifications of the transmission ML-EM algorithm [7]-[9] as well as algorithms that directly optimize the penalizedlikelihood objective [3], [10]-[13] have been introduced. Some of these algorithms seem to converge rapidly in the convex case.

However, up until now, no practically realizable monotonic (or convergent) algorithm has been found for the penalizedlikelihood problem when the objective is not convex. The negative log likelihood is nonconvex when there are background counts in the data. This is unavoidable in PET and SPECT due to the accidental coincidences in PET and emission crosstalk ${ }^{1}$ in SPECT. The assumption of no background counts may be reasonable in X-ray $\mathrm{CT}$.

In this paper, we present a new algorithm which is guaranteed to be monotonic, even when the objective function is nonconvex. This algorithm depends on paraboloidal surrogate functions for the log likelihood, which transform the problem into a simpler quadratic optimization problem at each iteration. The transformed problem at each iteration is similar to a penalized weighted least squares (PWLS) problem and, thus, has a familiar and simple form. This quadratic problem need not be solved exactly. An algorithm that monotonically decreases the surrogate function suffices. Since evaluating the gradient and Hessian of the surrogate function is much less costly, the CPU time per iteration is greatly reduced as compared to algorithms that directly attempt to minimize the objective function, such as coordinate descent (CD). Remarkably, the convergence rate is comparable to other direct algorithms. For nonconvex

\footnotetext{
${ }^{1}$ Even though different photon energies are used in simultaneous emission/transmission SPECT imaging, some emission events are recorded in the transmission energy window, due to Compton scatter and finite energy resolution.
} 
objective functions, monotonicity alone does not guarantee convergence to the global minimizer when local minima exist, but it does ensure that the estimates do not diverge, since the likelihood is bounded. Whether the transmission log likelihood has multiple local minima is an open question.

The surrogate or substitute function idea is not new to the tomographic reconstruction area. EM algorithms can be viewed as providing a surrogate function for the log-likelihood function by means of a statistically more informative complete data set which is unobservable [5]. The conditional expectation of the log-likelihood function for this new space is often easier to maximize, having a closed form for the emission case. This statistical construction of surrogate functions is somewhat indirect and seems to yield a limited selection of choices. De Pierro has developed surrogate functions for nonnegative least squares problems, based solely on convexity arguments, rather than statistics [14]. Our proposed approach is similar in spirit.

The EM algorithm did not result in a closed-form M-step for the transmission case [6], so direct minimization of the objective function became more attractive. Cyclic Newtonian $\mathrm{CD}(\mathrm{CD}, \mathrm{NR})$ [11] has been used effectively in transmission tomography. However, CD based on Newton's iteration for each pixel is not guaranteed to be monotonic. Furthermore, an iteration of Newton-based CD requires at least $M$ exponentiations and $17 M$ floating point operations, ${ }^{2}$ where $M$ is the (very large) number of nonzero elements in the system matrix $\boldsymbol{A}$ in (1) below. These exponentiations and floating point operations constitute a significant fraction of the CPU time per iteration. Recently, Zheng et al. introduced a functional substitution (FS) method [15], [16] which is proven to be monotonic for transmission scans with no background counts $\left[r_{i}=0\right.$ in (1) below]. Like CD, the FS algorithm cyclically updates the coordinates of the image vector, i.e., the attenuation map values for each pixel. However, instead of minimizing the original complex objective function with respect to each parameter, the FS algorithm minimizes a one-dimensional (1-D) parabolic surrogate function. The minimization of the surrogate is guaranteed to monotonically decrease the original objective function if the derivative of the negative $\log$ likelihood is concave (which is true when $r_{i}=0$ ) [15], [16]. On the other hand, the FS algorithm requires at least $2 M$ exponentiations and $17 M$ floating point operations ${ }^{3}$ per iteration, which means that the guarantee of monotonicity comes at a price of significantly increased computation time per iteration for that method. Furthermore, the FS algorithm is not monotonic in the nonconvex case of interest in PET and SPECT, where $r_{i} \neq 0$.

De Pierro [17] has used a surrogate function for the penalty part of the penalized-likelihood problem for convex penalties. The surrogate function idea was also used in several algorithms which update a group of pixel values at a time, rather than a sequential update of each pixel. Examples of these types of algorithms are the convex algorithm of [18], which updates all pixels simultaneously, and the grouped coordinate ascent

\footnotetext{
${ }^{2}$ This can be reduced to $9 M$ floating point operations if the denominator terms are precomputed similarly to Section III-F in this paper.

${ }^{3}$ Precomputation of the denominator terms in FSCD would destroy monotonicity.
}

(GCA) algorithm of [12] and [15], which updates a subset of pixels at a time. The surrogate functions used in these algorithms were obtained using De Pierro's convexity trick [17] to form a separable function that is easier to minimize than the nonseparable original objective function. The convergence rates per iteration decrease due to the higher curvature of these surrogate functions, but these algorithms require less computation per iteration as compared to single CD [11] and are parallelizable. Furthermore, it is trivial to impose the nonnegativity constraint with an additively separable surrogate function [12].

In this paper, we propose the use of a global surrogate function for the original objective function which is not separable, but has a simple quadratic form. The method is based on finding 1-D parabolic functions that are tangent to and lie above each of the terms in the log likelihood, similar to Huber's method for robust linear regression [19]. Whereas Huber considered strictly convex cost functions, we extend the method to derive provably monotonic algorithms, even for nonconvex negative log-likelihood functions. Remarkably, these algorithms require less CPU time to converge than the fastest algorithm introduced before (GCA of [12]) and, as an additional advantage, they are proven to be monotonic. We call the new approach to image reconstruction the paraboloidal surrogates (PS) method.

In the rest of this paper, we describe the problem, develop the new algorithm, and present representative performance results on real PET transmission data.

\section{THE PROBLEM}

The measurements in a photon-limited application, such as PET or SPECT, are modeled appropriately as Poisson random variables. In transmission tomography, the means of the prompt coincidences are related exponentially to the projections (or line integrals) of the attenuation map through Beer's law [6]. In addition, the measurements are contaminated by extra background counts, due mostly to random coincidences and scatter in PET and emission crosstalk in SPECT. Thus, it is realistic to assume the following model:

$$
y_{i} \sim \operatorname{Poisson}\left\{b_{i} e^{-[\boldsymbol{A} \mu]_{i}}+r_{i}\right\}, \quad i=1, \cdots, N
$$

where $N$ is the number of measurements, $\mu_{j}$ is the average linear attenuation coefficient in voxel $j$ for $j=1, \cdots, p$, and $p$ denotes the number of voxels. The notation $[\boldsymbol{A} \mu]_{i}=$ $\sum_{j=1}^{p} a_{i j} \mu_{j}$ represents the $i$ th line integral of the attenuation map $\mu$ and $\boldsymbol{A}=\left\{a_{i j}\right\}$ is the $N \times p$ system matrix. We assume that $\left\{b_{i}\right\},\left\{r_{i}\right\}$, and $\left\{a_{i j}\right\}$ are known nonnegative constants, where $r_{i}$ is the mean number of background events, $b_{i}$ is the blank scan factor, and $y_{i}$ represents the number of transmission events counted by the $i$ th detector (or detector pair in PET).

We seek to find a statistical estimate of the attenuation map $\mu$ which agrees with the data and is anatomically reasonable.

\footnotetext{
${ }^{4}$ The assumption that the background counts $r_{i}$ are known nonnegative constants is an approximation. In PET, we estimate the $r_{i}$ 's by smoothing the delayed coincidences from the transmission scan [20]. Alternatively, one can use time scaled delayed coincidences from a blank scan (which are less noisy due to longer scan times) as the $r_{i}$ factors [21] or use Bayesian estimation techniques to estimate the $r_{i}$ 's from delayed coincidences [3], [20].
} 
For this purpose, a natural approach is to use a likelihoodbased estimation strategy. The log-likelihood function for the independent transmission data is

$$
L(\mu)=\sum_{i=1}^{N}\left\{y_{i} \log \left(b_{i} e^{-[\boldsymbol{A} \mu]_{i}}+r_{i}\right)-\left(b_{i} e^{-[\boldsymbol{A} \mu]_{i}}+r_{i}\right)\right\}
$$

ignoring constant terms. The log likelihood depends on the parameter vector $\mu$ through its projections $[\boldsymbol{A} \mu]_{i}$ only and can be expressed in the following form:

$$
-L(\mu)=\sum_{i=1}^{N} h_{i}\left([A \mu]_{i}\right)
$$

where the contribution of the $i$ th measurement to the negative log likelihood is given by

$$
h_{i}(l) \triangleq\left(b_{i} e^{-l}+r_{i}\right)-y_{i} \log \left(b_{i} e^{-l}+r_{i}\right) .
$$

The proposed algorithm exploits the additive form of (2). Directly minimizing $-L(\mu)$ (maximum likelihood) results in a very noisy estimate $\hat{\mu}$ due to the ill-posed nature of the problem. However, it is well known that the attenuation map in the body consists of approximately locally homogeneous regions. This property has formed the basis of many segmentation methods for transmission scans [22]. Rather than applying hard segmentation, we add to the negative $\log$ likelihood a penalty term which encourages piecewise smoothness in the image, resulting in the penalized-likelihood image reconstruction formulation as given below:

$$
\hat{\mu}=\underset{\mu \geq 0}{\operatorname{argmin}} \Phi(\mu), \quad \Phi(\mu)=-L(\mu)+\beta R(\mu) .
$$

Our goal is to develop an algorithm for finding the minimizing $\hat{\mu}$ with minimal CPU time.

We consider roughness penalties $R(\mu)$ that can be expressed in the following very general form [17], [23]:

$$
R(\mu)=\sum_{k=1}^{K} \psi_{k}\left([\boldsymbol{C} \mu]_{k}\right)
$$

where the $\psi_{k}$ 's are potential functions acting as a norm on the soft constraints $\boldsymbol{C} \mu \approx 0$ and $K$ is the number of such constraints. The functions $\psi_{k}$ we consider are convex, symmetric, nonnegative, differentiable, and satisfy some more conditions that are listed in Section III-C. The $\beta$ in (4) is a parameter which controls the level of smoothness in the final reconstructed image. For more explanation of the penalty function, see [23].

The objective function defined in (4) is not convex when there are nonzero background counts $\left(r_{i} \neq 0\right)$ in the data. In this realistic case, there is no guarantee that there is a single global minimum. However, some practical algorithms exist that seem to work very well, yet none of them are proven to be monotonic. In this paper we introduce an algorithm that is monotonic, even when $\Phi$ is not convex. The new approach is based on successive paraboloidal surrogate functions and will be explained in the rest of the paper.

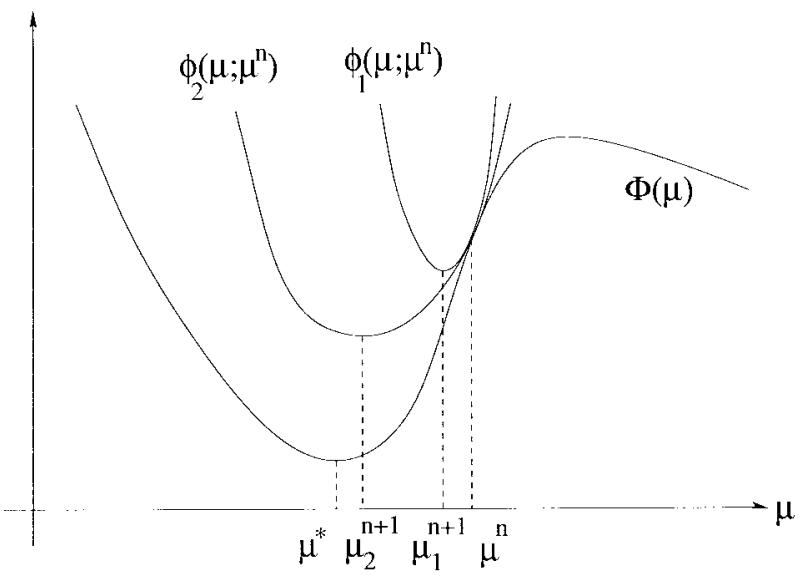

Fig. 1. One-dimensional illustration of the optimization transfer principle. Instead of minimizing $\Phi(\mu)$, we minimize the surrogate function $\phi\left(\mu ; \mu^{n}\right)$ at the $n$th iteration. Here, the surrogate function $\phi_{2}$ has a smaller curvature and is wider than $\phi_{1}$, thus, it has a bigger step size and hence a faster convergence rate to the local minimum $\mu^{*}$.

\section{Paraboloidal Surrogates Algorithms}

The penalized-likelihood objective function $\Phi(\mu)$ has a complex form that precludes analytical minimization. Thus, iterative methods are necessary for minimizing $\Phi(\mu)$. Our approach uses the optimization transfer idea proposed by De Pierro [14], [17], summarized as follows. Let $\mu^{n}$ be the attenuation map estimate after the $n$th iteration. We would like to find a surrogate function ${ }^{5} \phi\left(\mu ; \mu^{n}\right)$, which is easier to minimize or to monotonically decrease than $\Phi(\mu)$. This approach transforms the optimization problem into a simpler problem at each iteration, as illustrated in Fig. 1. The following "monotonicity condition on the surrogate function is sufficient to ensure that the iterates $\left\{\mu^{n}\right\}$ monotonically decrease $\Phi$

$$
\Phi(\mu)-\Phi\left(\mu^{n}\right) \leq \phi\left(\mu ; \mu^{n}\right)-\phi\left(\mu^{n} ; \mu^{n}\right), \quad \forall \mu \geq 0 .
$$

We restrict ourselves to differentiable surrogate functions, for which the following conditions are sufficient ${ }^{6}$ to ensure (6):

1) $\phi\left(\mu^{n} ; \mu^{n}\right)=\Phi\left(\mu^{n}\right)$

2) $\left.\frac{\partial \phi}{\partial \mu_{j}}\left(\mu ; \mu^{n}\right)\right|_{\mu=\mu^{n}}=\left.\frac{\partial \Phi}{\partial \mu_{j}}(\mu)\right|_{\mu=\mu^{n}}, \quad j=1, \cdots, p$

3) $\phi\left(\mu ; \mu^{n}\right) \geq \Phi(\mu)$, for $\mu \geq 0$.

Fig. 1 illustrates two surrogate functions that are tangent to the original objective at the current iterate and lie above it for all feasible values of the parameters.

The EM algorithm [6] provides a statistical method for constructing surrogate functions $\phi\left(\mu ; \mu^{n}\right)$, satisfying the above conditions. However, in the transmission tomography problem, the natural EM surrogate is difficult to minimize and leads to slow convergence. In this paper, we construct a simpler surrogate, using ordinary calculus rather than statistical techniques.

The log-likelihood function (2) has a certain kind of dependence on the parameters $\mu$, namely through their projections

\footnotetext{
${ }^{5}$ We use the notation $\phi\left(\mu ; \mu^{n}\right)$ to emphasize that the surrogate is a function of $\mu$ once $\mu^{n}$ is fixed and it changes for each $\mu^{n}$, following the $Q$ function notation of the EM algorithm [5].

${ }^{6}$ The second condition follows from the other two conditions for differentiable surrogate functions.
} 
$A \mu$. The negative log likelihood is the sum of individual functions $h_{i}$, each of which depends on a single projection only. We can exploit this form of the likelihood function by selecting a 1-D surrogate function for each of the 1-D $h_{i}$ functions in the projection $(l)$ domain. The overall sum of these individual 1-D functions will be an appropriate surrogate for the likelihood part of the objective.

Let $l_{i}^{n}=\left[A \mu^{n}\right]_{i}$ denote the estimate of the $i$ th line integral of the attenuation coefficient at the $n$th iteration. We choose the following quadratic form for the surrogate functions $q_{i}$ :

$$
q_{i}\left(l ; l_{i}^{n}\right) \triangleq h_{i}\left(l_{i}^{n}\right)+\dot{h}_{i}\left(l_{i}^{n}\right)\left(l-l_{i}^{n}\right)+\frac{1}{2} c_{i}\left(l_{i}^{n}\right)\left(l-l_{i}^{n}\right)^{2}
$$

where $c_{i}\left(l_{i}^{n}\right)$ is the curvature of the parabola $q_{i}$ and $\dot{h}$ denotes the first derivative of $h$. This construction ensures that $q_{i}\left(l_{i}^{n} ; l_{i}^{n}\right)=h_{i}\left(l_{i}^{n}\right)$ and $\dot{q}_{i}\left(l_{i}^{n} ; l_{i}^{n}\right)=\dot{h}_{i}\left(l_{i}^{n}\right)$ similar to (7). To ensure monotonicity, we must choose the curvatures to satisfy the following inequality at each iteration:

$$
h_{i}(l) \leq q_{i}\left(l ; l_{i}^{n}\right), \quad \text { for } l \geq 0 .
$$

After determining the parabolas, one can easily verify that the following function is a global surrogate function for the objective $\Phi(\mu)$ which satisfies the properties in (7)

$$
\phi\left(\mu ; \mu^{n}\right)=Q\left(\mu ; \mu^{n}\right)+\beta R(\mu)
$$

where

$$
\begin{aligned}
Q\left(\mu ; \mu^{n}\right) \triangleq & \sum_{i=1}^{N} q_{i}\left([A \mu]_{i} ; l_{i}^{n}\right) \\
= & \Phi\left(\mu^{n}\right)+\boldsymbol{d}_{h}\left(l^{n}\right)^{\prime} A\left(\mu-\mu^{n}\right) \\
& +\frac{1}{2}\left(\mu-\mu^{n}\right)^{\prime} \boldsymbol{A}^{\prime} D\left(c_{i}\left(l_{i}^{n}\right)\right) \boldsymbol{A}\left(\mu-\mu^{n}\right)
\end{aligned}
$$

where the column vector $\boldsymbol{d}_{h}\left(l^{n}\right) \triangleq\left[\dot{h}_{i}\left(l_{i}^{n}\right)\right]_{i=1}^{N}, \boldsymbol{x}^{\prime}$ denotes the transpose of $\boldsymbol{x}$ and $\boldsymbol{D}\left(c_{i}\left(l_{i}^{n}\right)\right)$ is the $N \times N$ diagonal matrix with diagonal entries $c_{i}\left(l_{i}^{n}\right)$ for $i=1, \cdots, N$.

The surrogate function $\phi\left(\mu ; \mu^{n}\right)$ in (10) consists of the sum of a paraboloid (i.e., a quadratic form) and the convex penalty term. An algorithm that decreases the function $\phi$ will also monotonically decrease the objective function if the inequality in (9) holds. The general paraboloidal surrogates (PS) method can be outlined as follows:

for each iteration $n$

$$
\begin{aligned}
& \text { determine } c_{i}\left(l_{i}^{n}\right) \text { and consequently } \phi\left(\mu ; \mu^{n}\right) \\
& \text { find a } \mu^{n+1} \geq 0 \text { that decreases (or minimizes) } \\
& \phi\left(\mu ; \mu^{n}\right)
\end{aligned}
$$

end.

The key design choices in the general method outlined above are the following:

1) the different ways of choosing the curvatures $c_{i}\left(l_{i}^{n}\right)$ 's which would satisfy (9);

2) the algorithm to monotonically decrease $\phi\left(\mu ; \mu^{n}\right)$ defined in (10) for $\mu \geq 0$.

Each combination of choices leads to a different algorithm, as we elaborate in the following sections.

\section{A. Maximum Curvature}

A natural choice for $c_{i}\left(l_{i}^{n}\right)$ is the maximum second derivative in the feasible region for the projections. This maximum curvature ensures that (9) holds, which follows from the generalized mean value theorem for twice differentiable functions $[24$, p. 228]. The feasible region for the projections is $[0, \infty)$, due to the nonnegativity constraint. Hence, the choice

$$
c_{i}\left(l_{i}^{n}\right)=\max _{l \in[0, \infty)}\left\{\ddot{h}_{i}(l)\right\}
$$

is guaranteed to satisfy (9). We show in Appendix A that the closed-form expression for $c_{i}\left(l_{i}^{n}\right)$ is

$$
c_{i}\left(l_{i}^{n}\right)=\left[\left(1-\frac{y_{i} r_{i}}{\left(b_{i}+r_{i}\right)^{2}}\right) b_{i}\right]_{+}
$$

where $[x]_{+}=x$ for $x>0$ and zero otherwise. Thus, it is trivial to compute the $c_{i}\left(l_{i}^{n}\right)$ terms in this case. The choice (14) for the curvature $c_{i}\left(l_{i}^{n}\right)$ does not depend on the iteration $n$, so it is a constant. We refer to this choice as the maximum curvature (PS,M,CD).

Having specified the curvatures $\left\{c_{i}\left(l_{i}^{n}\right)\right\}$, the paraboloidal surrogate $Q\left(\mu ; \mu^{n}\right)$ in (12) is now fully determined. Next, we need an algorithm that decreases or minimizes the surrogate function $\phi\left(\mu ; \mu^{n}\right)$.

\section{B. Algorithms for Minimizing the Paraboloidal Surrogate}

In the absence of the nonnegativity constraint, in principle, one could minimize the surrogate function $\phi\left(\mu ; \mu^{n}\right)$ over $\mu$ by zeroing its gradient. The column gradient of $\phi\left(\mu ; \mu^{n}\right)$ with respect to $\mu$ is given by

$$
\begin{aligned}
& \nabla_{\mu} \phi\left(\mu ; \mu^{n}\right) \\
& \quad=\boldsymbol{A}^{\prime} \boldsymbol{d}_{h}\left(l^{n}\right)+\boldsymbol{A}^{\prime} \boldsymbol{D}\left(c_{i}\left(l_{i}^{n}\right)\right) \boldsymbol{A}\left(\mu-\mu^{n}\right)+\beta \nabla R(\mu) .
\end{aligned}
$$

If $R(\mu)$ is a quadratic form, i.e., $R(\mu)=\frac{1}{2} \mu^{\prime} R \mu$, then we can analytically zero the gradient, yielding the iteration

$$
\mu^{n+1}=\mu^{n}-\left[\boldsymbol{A}^{\prime} \boldsymbol{D}\left(c_{i}\left(l_{i}^{n}\right)\right) \boldsymbol{A}+\beta \boldsymbol{R}\right]^{-1} \nabla_{\mu} \Phi\left(\mu^{n}\right) .
$$

There are three problems with the above iteration. It does not enforce the nonnegativity constraint, the matrix inverse is impractical to compute exactly, and it is limited to quadratic penalty functions. To overcome these limitations, we instead apply a monotonic CD iteration to decrease $\phi\left(\mu ; \mu^{n}\right)$.

\section{Coordinate Descent Applied to the Surrogate Function}

To apply CD to monotonically decrease the surrogate function $\phi\left(\mu ; \mu^{n}\right)$, we need a quadratic function that majorizes (lies above) the function $\phi\left(\mu ; \mu^{n}\right)$ at each pixel. We treat the likelihood part and the penalty part separately. Let

$$
\hat{Q}_{j}^{n}\left(\mu_{j}\right) \triangleq Q\left(\left[\hat{\mu}_{1}, \cdots, \hat{\mu}_{j-1}, \mu_{j}, \hat{\mu}_{j+1}, \cdots, \hat{\mu}_{p}\right] ; \mu^{n}\right)
$$

and

$$
\hat{R}_{j}^{\mathrm{o}}\left(\mu_{j}\right) \triangleq R\left(\left[\hat{\mu}_{1}, \cdots, \hat{\mu}_{j-1}, \mu_{j}, \hat{\mu}_{j+1}, \cdots, \hat{\mu}_{p}\right]\right)
$$

where $\hat{\mu}$ denotes the current estimate of the parameter $\mu$. Then we must select curvatures $d_{j}^{n}$ and $\hat{p}_{j}$ that satisfy the following:

$$
\hat{Q}_{j}^{n}\left(\mu_{j}\right)=Q\left(\hat{\mu} ; \mu^{n}\right)+\dot{Q}_{j}^{n}(\hat{\mu})\left(\mu_{j}-\hat{\mu}_{j}\right)+\frac{1}{2} d_{j}^{m}\left(\mu_{j}-\hat{\mu}_{j}\right)^{2}
$$


and

$$
\hat{R}_{j}^{\mathrm{o}}\left(\mu_{j}\right) \leq \hat{R}_{j}\left(\mu_{j}\right), \quad \forall \mu_{j} \geq 0
$$

where

$$
\hat{R}_{j}\left(\mu_{j}\right) \triangleq R(\hat{\mu})+\dot{R}_{j}(\hat{\mu})\left(\mu_{j}-\hat{\mu}_{j}\right)+\frac{1}{2} \hat{p}_{j}\left(\mu_{j}-\hat{\mu}_{j}\right)^{2}
$$

where $\hat{Q}_{j}^{n}\left(\mu_{j}\right)$ and $\hat{R}_{j}^{\mathrm{o}}\left(\mu_{j}\right)$ are treated as functions of $\mu_{j}$ only. Equality is achievable in (17) since the likelihood surrogate $\hat{Q}_{j}^{n}\left(\mu_{j}\right)$ is quadratic. For the penalty part $\hat{R}_{j}^{\circ}\left(\mu_{j}\right)$, we must find a quadratic function $\hat{R}_{j}\left(\mu_{j}\right)$ that lies above it, by appropriate choice of $\hat{p}_{j}$, as considered below.

The derivative of the likelihood surrogate parabola at $\hat{\mu}_{j}$ is [from (11)]

$$
\left.\dot{Q}_{j}^{n}(\hat{\mu}) \triangleq \frac{\partial}{\partial \mu_{j}} \hat{Q}_{j}^{n}\left(\mu_{j}\right)\right|_{\mu_{j}=\hat{\mu}_{j}}=\sum_{i=1}^{N} a_{i j} \dot{q}_{i}\left(\hat{l}_{i}\right)
$$

where from (8)

$$
\dot{q}_{i}\left(\hat{l}_{i}\right)=\dot{h}_{i}\left(l_{i}^{n}\right)+c_{i}\left(l_{i}^{n}\right)\left(\hat{l}_{i}-l_{i}^{n}\right)
$$

where $\hat{l}_{i}=\sum_{i=1}^{N} a_{i j} \hat{\mu}_{j}$ and

$$
\dot{h}_{i}(l)=\left(\frac{y_{i}}{b_{i} e^{-l}+r_{i}}-1\right) b_{i} e^{-l} .
$$

From (8) and (11), the curvature of the parabola $\hat{Q}_{j}^{n}\left(\mu_{j}\right)$ is obviously

$$
d_{j}^{n} \triangleq \sum_{i=1}^{N} a_{i j}^{2} c_{i}\left(l_{i}^{n}\right)
$$

From (5), the derivative of the penalty part at $\hat{\mu}_{j}$ is

$$
\left.\dot{R}_{j}(\hat{\mu}) \triangleq \frac{\partial}{\partial \mu_{j}} \hat{R}_{j}^{\circ}\left(\mu_{j}\right)\right|_{\mu_{j}=\hat{\mu}_{j}}=\sum_{k=1}^{K} c_{k j} \dot{\psi}_{k}\left([\boldsymbol{C} \hat{\mu}]_{k}\right) .
$$

We must obtain a parabolic surrogate $\hat{R}_{j}\left(\mu_{j}\right)$ that satisfies (18). We assume the potential functions $\psi_{k}(\cdot)$ satisfy the following conditions.

1) $\psi$ is symmetric.

2) $\psi$ is everywhere differentiable (and therefore continuous).

3) $\dot{\psi}(t)=d / d t \psi(t)$ is nondecreasing (and hence $\psi$ is convex).

4) $\omega_{\psi}(t) \triangleq \dot{\psi}(t) / t$ is nonincreasing for $t \geq 0$.

5) $\omega_{\psi}(0)=\lim _{t \rightarrow 0} \dot{\psi}(t) / t$ is finite and nonzero, i.e., $0<$ $\omega_{\psi}(0)<\infty$.

In the context of robust regression, Huber showed ([19, Lemma 8.3, p. 184], [23]) that for potential functions $\psi_{k}$ that satisfy the conditions above, we can find a parabola $\hat{\psi}_{k}(t)$ that lies above $\psi_{k}(t), \forall t \in \mathbb{R}$. This parabola $\hat{\psi}_{k}(t)$ is tangent to the potential function at the current point $\hat{t}_{k} \triangleq[C \hat{\mu}]_{k}$ and at $-\hat{t}_{k}$ and has the curvature $\omega_{\psi_{k}}\left(\hat{t}_{k}\right)$ where $\omega_{\psi}(\cdot)$ was defined above. The surrogate parabola is given by

$$
\hat{\psi}_{k}(t)=\psi_{k}\left(\hat{t}_{k}\right)+\dot{\psi}\left(\hat{t}_{k}\right)\left(t-\hat{t}_{k}\right)+\frac{1}{2} \omega_{\psi_{k}}\left(\hat{t}_{k}\right)\left(t-\hat{t}_{k}\right)^{2}
$$

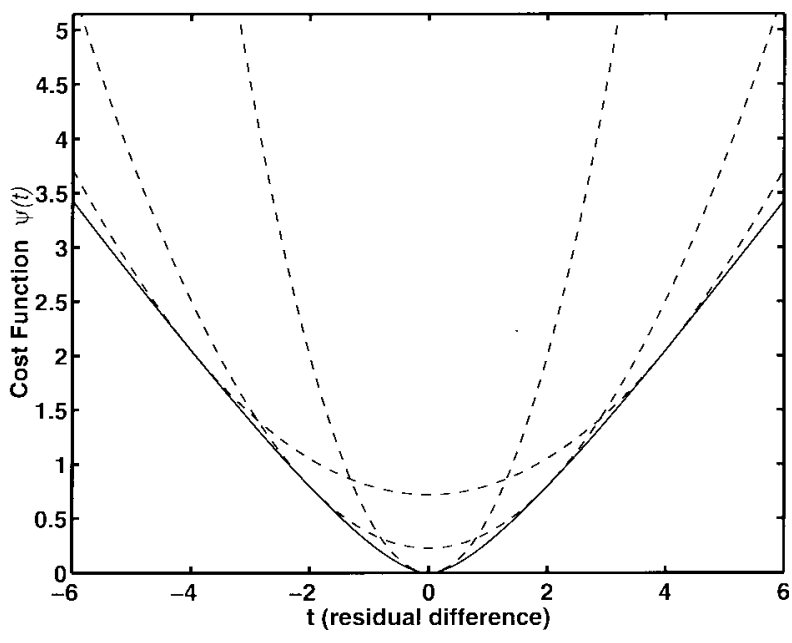

Fig. 2. Illustration of the tangent parabolas lying above a potential function.

and is illustrated in Fig. 2. Thus, the following is a surrogate parabola for the penalty part of the objective function:

$$
\hat{R}_{j}\left(\mu_{j}\right)=\left.\sum_{k=1}^{K} \hat{\psi}_{k}\left([\boldsymbol{C} \mu]_{k}\right)\right|_{\mu_{m}=\hat{\mu}_{m}, \quad \forall m \neq j} .
$$

The curvature of the parabola $\hat{R}_{j}\left(\mu_{j}\right)$ is

$$
\hat{p}_{j} \triangleq \sum_{k=1}^{K} c_{k j}^{2} \omega_{\psi_{k}}\left([\boldsymbol{C} \hat{\mu}]_{k}\right)
$$

Combining the above surrogate parabolas (17) and (23), the minimization step of the CD for pixel $j$ is simply

$$
\begin{aligned}
\hat{\mu}_{j}^{\text {new }} & =\underset{\mu_{j} \geq 0}{\operatorname{argmin}} \hat{Q}_{j}^{n}\left(\mu_{j}\right)+\beta \hat{R}_{j}\left(\mu_{j}\right) \\
& =\left[\hat{\mu}_{j}-\frac{\dot{Q}_{j}^{n}(\hat{\mu})+\beta \dot{R}_{j}(\hat{\mu})}{d_{j}^{n}+\beta \hat{p}_{j}}\right]_{+} .
\end{aligned}
$$

This is an update that monotonically decreases the value of $\phi\left(\cdot ; \mu^{n}\right)$ and consequently the value of $\Phi(\cdot)$. One iteration is finished when all pixels are updated via (25) in a sequential order. We usually update the paraboloidal surrogate function after one iteration of $\mathrm{CD}$, but one could also perform more than one CD iteration per surrogate. We call this method the paraboloidal surrogates CD (PSCD) method.

The PSCD algorithm with the curvatures obtained from (14) is outlined in Table I. In this table, the algorithm flow is given for the general case where $c_{i}\left(l_{i}^{n}\right)$ may change at each iteration. However, the curvatures $c_{i}\left(l_{i}^{n}\right)$ given in (35) in Table I are constant throughout the iterations. If one uses fixed $c_{i}\left(l_{i}^{n}\right)$ values which do not depend on $n$ as in (35), then the $d_{j}^{m}$ terms can be precomputed and the algorithm should be reorganized to take this computational advantage into account.

Another computational advantage of curvatures that do not depend on the iterations is as follows. If we define $\tilde{q}_{i}=\dot{q}_{i} / \sqrt{c_{i}}$ and $\tilde{a}_{i j}=a_{i j} \sqrt{c_{i}}$, then the update in (38) will be simplified to

$$
\tilde{q}_{i}:=\tilde{q}_{i}+\tilde{a}_{i j}\left(\mu_{j}^{\text {work }}-\hat{\mu}_{j}\right)
$$


TABLE I

Algorithm Outline for a Paraboloidal Surrogates Algorithm with Coordinate Descent (PSCD). The Curvature Choice Shown Here Is the Maximum Second Derivative

$$
\begin{aligned}
& \text { Initialize: } \hat{\mu}=\operatorname{rBP}\left\{\log \left(b_{i} /\left(y_{i}-r_{i}\right)\right)\right\}_{i=1}^{N} \text { and } \hat{l}_{i}-\sum_{j=1}^{p} a_{i j} \hat{\mu}_{j}, \forall i-1, \ldots, N \\
& \text { for each iteration } n=0, \ldots \text {, Niter }-1 \\
& \dot{q}_{i}=\dot{h}_{i}-\left(\frac{y_{i}}{b_{i} e^{-\bar{l}_{i}}+r_{i}}-1\right) b_{i} e^{\hat{l}_{i}}, \text { for } i-1, \ldots, N \\
& c_{i}=\max _{i \geq 0} \ddot{h}_{i}(l)=\left[\left(1-\frac{y_{i} r_{i}}{\left(b_{i}+r_{i}\right)^{2}}\right) b_{i}\right], \text { for } i-1, \ldots, N \\
& c_{i}:= \begin{cases}c_{i}, & c_{i}>\epsilon \\
\epsilon, & c_{i} \leq \epsilon\end{cases}
\end{aligned}
$$

repeat one or more times for $j=1, \ldots, p$

$$
\begin{gathered}
\dot{Q}_{j}=\sum_{i=1}^{N} a_{i j} \dot{q}_{i}, \quad d_{j}=\sum_{i=1}^{N} a_{i j}^{2} c_{i} \\
\mu_{j}^{(j \mathrm{ld}}-\hat{\mu}_{j}
\end{gathered}
$$

for a couple sub-iterations

$$
\hat{\mu}_{j}:=\left[\hat{\mu}_{j}-\frac{\left.\dot{Q}_{j}+d_{j}\left(\hat{\mu}_{j}-\mu_{j}^{\text {old }}\right)+\beta \sum_{k=1}^{K} c_{k j} \dot{\psi}(\boldsymbol{C} \hat{\mu}]_{k}\right)}{\left.d_{j}+\beta \sum_{k, 1}^{K} c_{k j}^{2} \omega_{\psi_{k}}(\boldsymbol{C} \hat{\mu}]_{k}\right)}\right],
$$

end

$$
\dot{q}_{i}:=\dot{q}_{i}+a_{i j} c_{i}\left(\hat{\mu}_{j}-\mu_{j}^{\text {old }}\right) \forall i \text { s.t. } a_{i j} \neq 0
$$

end

end

$$
\hat{l}_{i}:-\hat{l}_{i}+\frac{\dot{q}_{i}-\dot{h}_{i}}{c_{i}}, \text { for } i-1, \ldots, N
$$

end

which decreases the computation time devoted to back and forward projections per iteration by about $20 \%$ for implementations using precomputed system matrices. Equations (37) and (39) should also be modified to use the new variables. We have not implemented this faster version for this paper.

The algorithm in Table I requires roughly double the floating point operations required for one forward and one backprojection per iteration. The gradient of the original log likelihood with respect to the projections $\left\{\dot{h}_{i}\left(l_{i}^{n}\right)\right\}_{i=1}^{N}$ and the curvature terms $c_{i}\left(l_{i}^{n}\right)$ are computed only once per iteration. ${ }^{7}$ The gradient of the surrogate paraboloid uses $\dot{q}_{i}$ terms which can be updated easily, as shown in (38) in the algorithm. This implementation does not update the projections $\hat{l}_{i}$ after each pixel update, since they are only needed in the outer loop (34). The projections are computed in (39) after updating all pixels. The update (39) requires $c_{i}^{n}>0$ to work. In (36), we constrain the curvature value to some small value $\epsilon>0$ (which obviously does not hurt monotonicity) so that (39) can be evaluated for all $i=1, \cdots, N$. However, $\epsilon$ should not be very small, since it will cause undesirable numerical precision errors. Storage requirements are also modest for the proposed algorithm. A single copy of the image and four sinograms for

\footnotetext{
${ }^{7}$ In contrast to the PSCD algorithm, when coordinate descent (CD,NR) is applied to the original objective function, new gradients and curvatures must be computed after each pixel is updated. These computations involve expensive exponentiations and floating point operations which increase the $\mathrm{CPU}$ time required for original $\mathrm{CD}$.
}

$\hat{l}_{i}, c_{i}, \dot{h}_{i}$, and $\dot{q}_{i}$ need to be stored in addition to data vectors $y_{i}, b_{i}, r_{i}$.

In the following, we discuss the convergence rate of the algorithm, which provides motivation for obtaining better curvatures.

\section{Convergence and Convergence Rate}

In the absence of background events, i.e., when $r_{i}=$ 0 , the penalized-likelihood objective $\Phi$ is convex and our proposed PSCD algorithm is globally convergent. This is a fairly straightforward consequence of the proof in [25] for the convergence of SAGE, so we omit the details.

However when $r_{i} \neq 0$, little can be said about global convergence due to the possibility that there are multiple minima or a continuous region of minima. Our practical experience suggests that local minima are either unlikely to be present, or are quite far from reasonable starting images, since all experiments with multiple initializations of the algorithm yielded the same limit within numerical precision. The PSCD algorithm is monotonic, even with the nonconvex objective function. One can easily show that every fixed point of the algorithm is a stationary point of the objective function and vice versa. Thus, it is comforting to know that the algorithm will converge to a local minimum and will not blow up.

The convergence rate of the proposed algorithm with the maximum curvature choice is suboptimal. The curvatures 
$c_{i}\left(l_{i}^{n}\right)$ are too conservative and the paraboloids are unnecessarily narrow. Intuitively, one can deduce that smaller $c_{i}\left(l_{i}^{n}\right)$ values will result in faster convergence. The reason for this is that the lower the curvature, the wider the paraboloid and the bigger the step size, as can be seen in Fig. 1. To verify this intuition, we analyze the convergence rate of the algorithm. For simplicity, we assume that a quadratic penalty is used in the reconstruction and that the surrogate function $\phi\left(\mu ; \mu^{n}\right)$ (10) is minimized exactly.

Let $\hat{\mu}$ be the unconstrained minimizer of the original objective function. At step $n$, by zeroing the gradient of (10) we get the simple Newton-like update in (16). By the Taylor series, for $\mu^{n} \approx \hat{\mu}$, we can approximate the gradient of the objective function as $\nabla \Phi\left(\mu^{n}\right) \approx \boldsymbol{H}(\hat{\mu})\left(\mu^{n}-\hat{\mu}\right)$ where $\boldsymbol{H}(\hat{\mu})$ is the Hessian of $\Phi$ at $\hat{\mu}$. Define $\boldsymbol{N}(c)=\boldsymbol{A}^{\prime} \boldsymbol{D}\left(c_{i}\right) \boldsymbol{A}+\beta \boldsymbol{R}$, then from (16)

$$
\begin{aligned}
\mu^{n+1}-\hat{\mu} & \approx \mu^{n}-\hat{\mu}-[\boldsymbol{N}(c)]^{-1} \boldsymbol{H}(\hat{\mu})\left(\mu^{n}-\hat{\mu}\right) \\
& =\left(I-[N(c)]^{-1} \boldsymbol{H}(\hat{\mu})\right)\left(\mu^{n}-\hat{\mu}\right) .
\end{aligned}
$$

This equation describes how the convergence rate of the proposed algorithm is affected by different $c_{i}$ choices. We use the results from [26] to evaluate the convergence rate. Let $\boldsymbol{N}\left(c^{1}\right)$ and $\boldsymbol{N}\left(c^{2}\right)$ be two matrices corresponding to curvature vectors $c^{1}$ and $c^{2}$, respectively, with $c_{i}^{1}<c_{i}^{2}, \forall i$. Then, obviously, $\boldsymbol{N}\left(c^{2}\right)-\boldsymbol{N}\left(c^{1}\right)$ is positive definite and it follows from [26, Lemma 1] that the algorithm corresponding to $c^{1}$ has a lower root-convergence factor and thus converges faster than the algorithm corresponding to $c^{2}$.

Therefore, to optimize the convergence rate, we would like the $c_{i}\left(l_{i}^{n}\right)$ values to be as small as possible while still satisfying (9). The optimal choice for the curvatures is the solution to the following constrained optimization problem for each $i$ :

$$
\begin{aligned}
c_{i}\left(l_{i}^{n}\right)=\min & \left\{c \geq 0: h_{i}(l) \leq h_{i}\left(l_{i}^{n}\right)+\dot{h}_{i}\left(l_{i}^{n}\right)\left(l-l_{i}^{n}\right)\right. \\
& \left.+\frac{1}{2} c\left(l-l_{i}^{n}\right)^{2} \forall l \geq 0\right\}
\end{aligned}
$$

This choice yields the fastest convergence rate while still guaranteeing monotonicity. In the following section, we discuss the solution to (27).

\section{E. Optimum Curvature}

The curvature that satisfies (27) is not trivial to find for general functions $h_{i}(\cdot)$. However, the marginal negative loglikelihood functions for each projection $\left[h_{i}\right.$ defined in (3)] in transmission tomography have some nice properties. We show the following in Appendix B. The parabola that is 1) tangent to $h_{i}$ at the current projection $l_{i}^{n}$ and 2) intersects $h_{i}$ at $l=0$ is guaranteed to lie above $h_{i}(l) \forall l \geq 0$. This claim is true only when the curvature $c_{i}\left(l_{i}^{n}\right)$ of $q_{i}$ is nonnegative. If the curvature obtained by the above procedure is negative, then we set $c_{i}\left(l_{i}^{n}\right)$ to zero. ${ }^{8}$ When $c_{i}\left(l_{i}^{n}\right)=0$, the $q_{i}$ function is the line which is tangent to the $h_{i}$ curve at the current projection value $l_{i}^{n}$.$$
\text { (36). }
$$

${ }^{8}$ In fact, any nonnegative $c_{i}\left(l_{i}^{n}\right)$ will ensure monotonicity, hence, the $\epsilon$ in
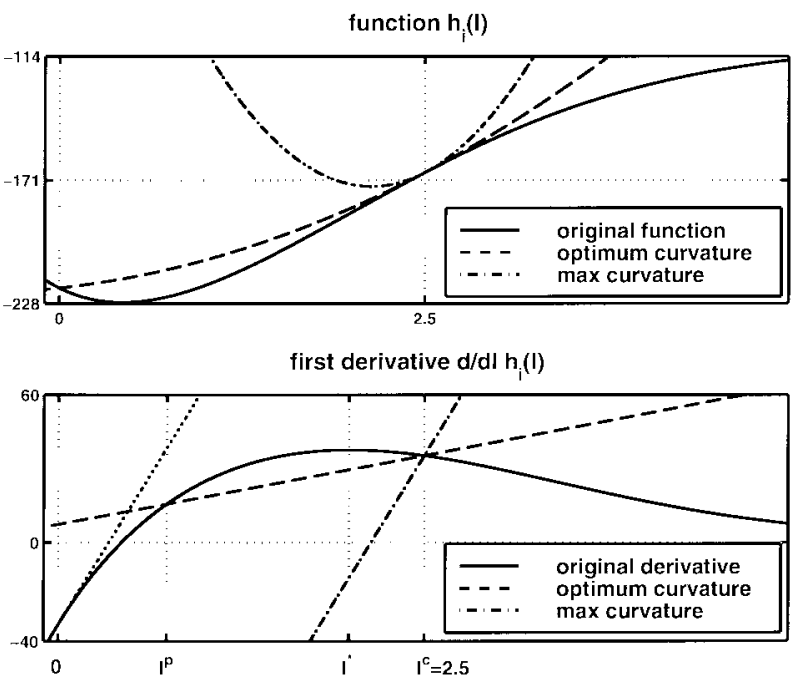

Fig. 3. This figure illustrates the optimum curvature and the maximum curvature surrogate functions and their derivatives for $b_{i}=100, y_{i}=70$, $r_{i}=5$, and $l_{i}^{n}=2.5$.

The curvature of the parabola described above is: ${ }^{9}$

$$
c_{i}\left(l_{i}^{n}\right)= \begin{cases}{\left[2 \frac{h_{i}(0)-h_{i}\left(l_{i}^{n}\right)+\dot{h}_{i}\left(l_{i}^{n}\right)\left(l_{i}^{n}\right)}{\left(l_{i}^{n}\right)^{2}}\right]_{+},} & l_{i}^{n}>0 \\ {\left[\ddot{h}_{i}(0)\right]_{+},} & l_{i}^{n}=0 .\end{cases}
$$

We prove in Appendix B that this curvature is the optimum curvature that satisfies (27). The nonnegativity constraint plays an important role in the proof. If nonnegativity is not enforced, the projections at an iteration may go negative and the curvature (28) will not guarantee monotonicity anymore. Fig. 3 illustrates this surrogate parabola with the optimum curvature (28). In Table I, the curvature computation in (35) should be changed to (28) to implement the PSCD method with the optimum curvature (PS,O,CD).

\section{F. Precomputed Curvature}

By relaxing the monotonicity requirement, we can develop faster yet almost always monotonic algorithms. We can do this by choosing curvatures $c_{i}\left(l_{i}^{n}\right)$ in (8) such that $\dot{h}_{i}(l)=$ $\dot{q}_{i}\left(l ; l_{i}^{n}\right)$, but $h_{i}(l) \approx q_{i}\left(l ; l_{i}^{n}\right)$, rather than requiring the inequality (9). In this case, the paraboloids are quadratic approximations to the log-likelihood function at each iteration. A reasonable choice for the curvatures is

$$
c_{i}=\ddot{h}_{i}\left(\log \frac{b_{i}}{y_{i}-r_{i}}\right)=\left(y_{i}-r_{i}\right)^{2} / y_{i} .
$$

The value $l_{i}^{\min }=\log \left(b_{i} /\left(y_{i}-r_{i}\right)\right)$ is the point that minimizes the $h_{i}$ function. These curvatures $c_{i}$ in (29) are close approximations to the second derivative of $h_{i}$ functions at the projection values $\boldsymbol{A} \hat{\mu}$ where $\hat{\mu}$ is the solution to the penalizedlikelihood problem [12]. This is called the fast denominator

\footnotetext{
${ }^{9}$ When $l_{i}^{n}$ is nonzero but small, due to numerical precision, (28) might turn out to be extremely large during computation. If $c_{i}\left(l_{i}^{n}\right)>\left[\ddot{h}_{i}(0)\right]_{+}$ (which theoretically should not happen, but practically happens due to limited precision), then we set $c_{i}\left(l_{i}^{n}\right)$ to be equal to the maximum second derivative $\left[\ddot{h}_{i}(0)\right]_{+}$which eliminates the problem.
} 
approach in [12], since it features a one-time precomputed approximation to the curvature that is left unchanged during the iterations so that the denominator terms $d_{j}^{n}$ (22) can be computed prior to iteration [similar to maximum curvature in (14)]. Computational benefits for iteration independent curvatures, as summarized in Section III-C, can be utilized. This approximation works well because we usually start the iterations with an FBP image $\mu^{0}$ where projections $\boldsymbol{A} \mu^{0}$ are usually close to $l^{\mathrm{min}}$. Nevertheless, unlike (28), monotonicity is not guaranteed with (29).

The PS method with the curvature (29) yields faster convergence than the other PS algorithms presented above. This method is related to the PWLS image reconstruction method [11], [27], but instead of making a one-time quadratic approximation to the log-likelihood function, the approximation is renewed at each iteration. Although the curvature of the paraboloid remains same, the gradient is changed to match the gradient of the original objective function at the current iterate. The nonnegativity constraint does not play an important role for the derivation, and this curvature may be used for algorithms where nonnegativity is not enforced. We refer to this curvature as precomputed curvature (PS,P,CD).

\section{RESULTS}

To assess the effectiveness and speed of the new PS algorithms, we present results using real PET data. We acquired a 15 -h blank scan $\left(b_{i}\right.$ 's) and a 12-min transmission scan data ( $y_{i}$ 's) using a Siemens/CTI ECAT EXACT 921 PET scanner with rotating rod transmission sources [28]. The phantom used was an anthropomorphic thorax phantom (Data Spectrum, Chapel Hill, NC). Delayed coincidence sinograms were collected separately in each scan. The blank and transmission scan delayed-coincidence sinograms were shown to be numerically $\operatorname{close}^{10}$ [21], so we used a time-scaled version of blank scan delayed coincidences as the $r_{i}$ factors, with no other processing. The projection space was 160 radial bins and 192 angles and the reconstructed images were $128 \times 128$ with 4.2-mm pixels. The system matrix $\left\{a_{i j}\right\}$ was computed by using 3.375-mm-wide strip integrals with 3.375-mm spacing, which roughly approximates the system geometry [4].

We performed reconstructions of the phantom by FBP as well as various penalized-likelihood methods. For the penalty term in PL reconstructions, we used the following function:

$$
R(\mu)=\frac{1}{2} \sum_{j=1}^{p} \sum_{k \in \mathcal{N}_{j}} w_{j k} \psi\left(\mu_{j}-\mu_{k}\right)
$$

which is a special case of (5). Here, $w_{j k}$ is normally equal to one for horizontal and vertical neighbors and $1 / \sqrt{2}$ for diagonal neighbors. We used the modified $w_{j k}$ 's described in [29] to achieve more uniform resolution. For the potential function, we used one of the edge-preserving nonquadratic cost functions that were introduced in [30]

$$
\psi(x)=\delta^{2}[|x / \delta|-\log (1+|x / \delta|)]
$$

\footnotetext{
${ }^{10}$ This is due to the fact that singles rate is mostly affected by transmission rods.
}

This function acts like a quadratic penalty for small differences in neighboring pixels and is close to the absolute value function for differences greater than $\delta$. This nonquadratic function penalizes sharp edges less than quadratic functions. We used $\delta=0.004 \mathrm{~cm}^{-1}$ chosen by visual inspection. In the final reconstructed image, the horizontal and vertical neighbor differences are less than this $\delta$ in homogeneous regions $(90 \%$ of all differences) which makes the curved part of the penalty effective in those regions. However, at edges for which the differences are greater than $\delta$, this penalty penalizes less than the quadratic one.

The PS algorithms described throughout this section are named using the following format: PS,,$C D$. PS stands for paraboloidal surrogates as the general framework for the algorithms and CD stands for coordinate descent applied to the surrogate function. The letter $\mathcal{C}$ in the format represents the curvature type $c_{i}\left(l_{i}^{n}\right)$. The types are $\mathrm{M}, \mathrm{O}$, and $\mathrm{P}$ for maximum second derivative curvature (14), optimum curvature (28), and precomputed curvature (29), respectively. The other algorithms we used for comparison in this section are as follows: the LBFGS, a constrained quasi-Newton algorithm [31]; CD,P,CD with precomputed denominators; CD,NR,CD with Newton-Raphson denominators [11], [12] applied to the objective function; and GD,P, grouped descent with precomputed denominators [12].

Fig. 4 shows images reconstructed by FBP and statistical methods from a 12-min scan. For comparison, an FBP reconstruction of a 7-h scan is also shown. Qualitatively, the statistical reconstruction looks better than the FBP image, having less noise and more uniform homogeneous regions. However, our focus here is not the image quality but the amount of time it takes the algorithms to converge to the minimizer image. Nevertheless, improved emission image quality is our ultimate goal. Statistical methods for transmission reconstruction yield better ACF's, as compared to conventional methods and result in better emission images. Our goal here is to speed up and stabilize statistical methods to make them usable routinely in clinic.

Fig. 5 shows that the proposed PSCD algorithms decreased $\Phi$ almost as much per iteration as the CD algorithm applied to $\Phi$ directly. This result is important because it shows that the surrogate paraboloids (especially with the optimum curvature) closely approximate the original log likelihood. More importantly, in Fig. 6 the PSCD algorithms are seen to be much faster than CD in terms of the actual CPU time. ${ }^{11}$ One of the main overhead costs in $\mathrm{CD}$ is the computation of the loglikelihood gradient term after each pixel change [12]. In the PSCD algorithm, the gradient of the surrogate function $\left(\dot{q}_{i}\right.$ 's) can be computed (updated) by a single multiplication (20). The maximum curvature method introduced in Section III-A precomputes the denominator terms $\left(d_{j}^{n}\right)$ for the likelihood part since $c_{i}\left(l_{i}^{n}\right)$ 's do not depend on the iterations. However, these $c_{i}\left(l_{i}^{n}\right)$ 's are much larger than the optimal curvatures, so more iterations are required for PS,M,CD to converge than for PS,O,CD.

\footnotetext{
${ }^{11}$ All CPU times are recorded on a DEC 600 5-333-MHz workstation with
} compiler optimization enabled. 


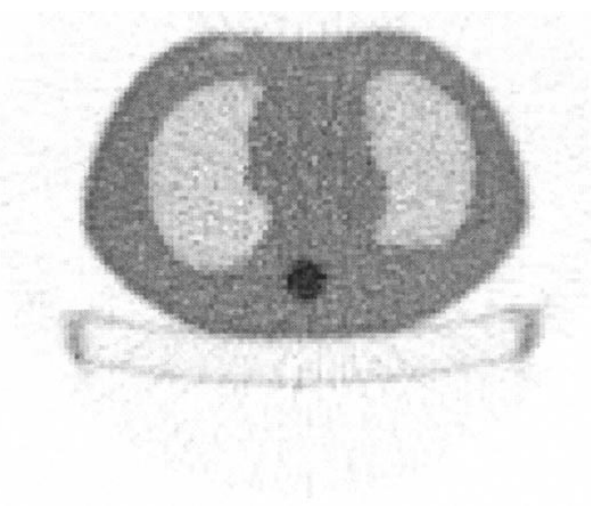

(a)

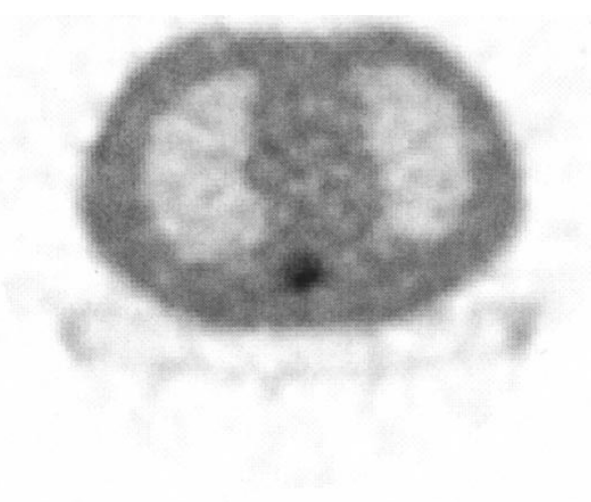

(b)

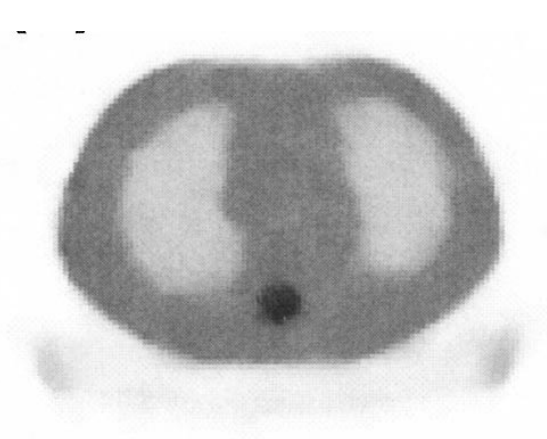

(c)

Fig. 4. (a) FBP reconstruction of phantom data from 7-h transmission scan. (b) FBP reconstruction from 12-min transmission scan. (c) Penalized-likelihood reconstruction from 12-min transmission scan using 12 iterations of the optimum curvature PSCD algorithm.

We also compared the PSCD algorithms to the generalpurpose constrained quasi-Newton algorithm (LBFGS) [31] in Figs. 5 and 6. Although the LBFGS algorithm takes about 25\% less CPU time $(0.88 \mathrm{~s})$ per iteration than PSCD algorithms, it did not converge as fast as the proposed algorithms. This shows that the algorithms such as PSCD, which are tailored to our specific problem, converge faster than the general-purpose quasi-Newton method.

In Fig. 7, we consider the fastest previous algorithm we know of (i.e., GD with $3 \times 3$ groups with a precomputed denominator [12] and compare it to the fastest PS algorithms. The PSCD with precomputed curvatures (PS,P,CD) (introduced in Section III-F) requires slightly less CPU time than GD,P to

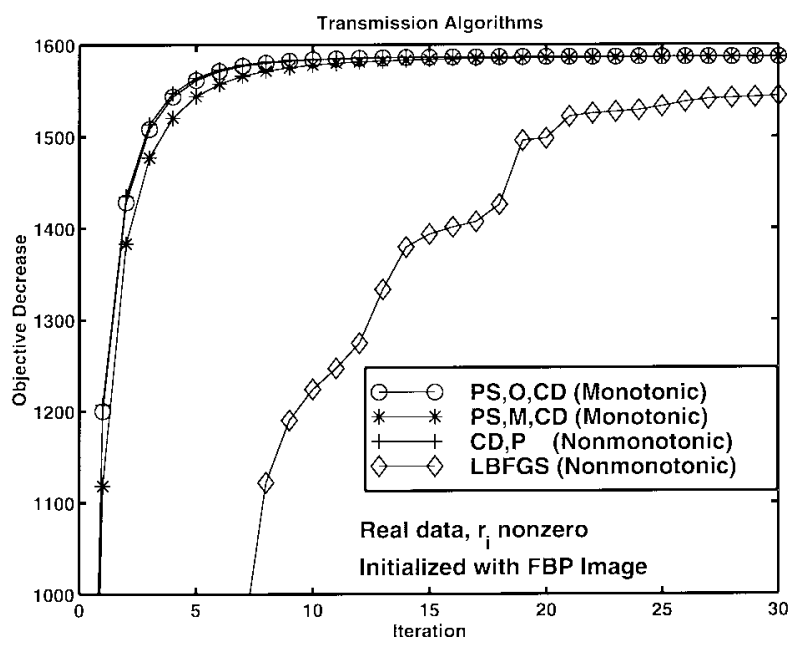

Fig. 5. Comparison of objective function decrease $\Phi\left(\mu^{0}\right)-\Phi\left(\mu^{n}\right)$ versus iteration number $n$ of monotonic PS methods with CD and LBFGS methods for real phantom data.

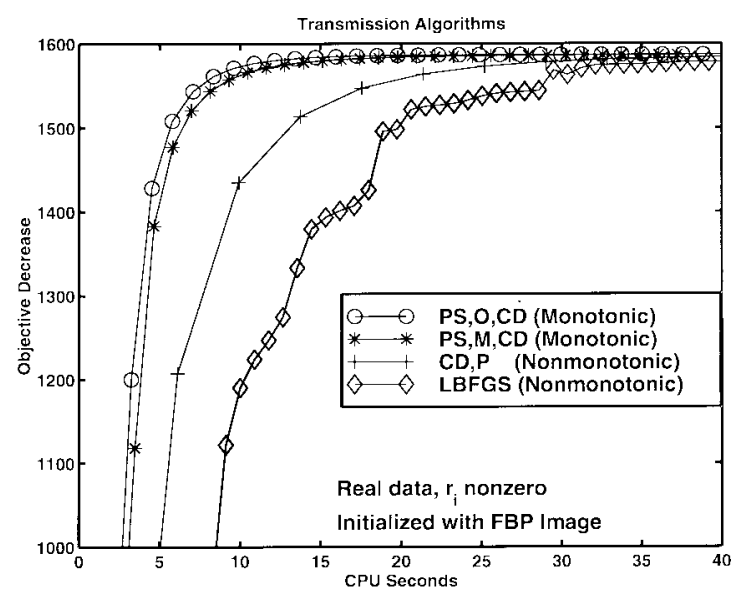

Fig. 6. Same as Fig. 5, but $x$ axis is CPU seconds on a DEC AlphaStation $6005-333 \mathrm{MHz}$.

converge. Although the PS,P,CD algorithm is not provably monotonic, it is a reasonable approximation and we did not observe any nonmonotonicity in our practical experience, when initializing with an FBP image. The monotonic PS, O,CD method is shown in this plot as a baseline for comparison with Fig. 6.

In Fig. 8, we present the results of a transmission scan simulation with zero background counts $\left(r_{i}=0\right)$ and compare the monotonic PSCD algorithm with the functional substitution (FS) method of Zheng et al. [15], [16]. The FS algorithm is proven to be monotonic when $r_{i}=0$, in which case $h_{i}$ is convex. However, the FSCD method requires considerably more computation per iteration than both CD and PSCD. The plot in Fig. 8 shows that FSCD requires more CPU time than PSCD.

Table II compares the number of iterations and CPU seconds required to minimize the objective function by each method. The CPU times, ${ }^{12}$ floating point operations, and mem-

\footnotetext{
${ }^{12}$ The CPU times are computed on a DEC 600 5-333 MHz. We also compiled the code on a SUN Ultra 2 computer and got similar CPU time ratios for the algorithms. However, the ratios could differ on another architecture or with another compiler, due to cache size and pipelining differences.
} 
TABLE II

Comparison of CPU Times, Number of Iterations to Converge, Floating Point Operations and Memory Accesses for the PS Algorithms Versus CD, GD, and FS Methods. Convergence in This Table Means $\Phi\left(\mu^{0}\right)-\Phi\left(\mu^{n}\right)>0.999\left[\Phi\left(\mu^{0}\right)-\Phi\left(\mu^{*}\right)\right]$ Where $\Phi\left(\mu^{*}\right)$ Is the Smallest Objective Value Obtained in 30 Iterations Among All the Methods. The Floating Point Operations, and Memory Accesses Only in the Order of $M$ Are Shown for Each Method

\begin{tabular}{|c|c|c|c|c|c|c|c|}
\hline \multirow{2}{*}{$\begin{array}{l}\text { Real data, } r_{i} \neq 0 \\
\text { methods }\end{array}$} & \multicolumn{2}{|c|}{ monotonic } & \multicolumn{5}{|c|}{ nonmonotonic } \\
\hline & PS,M,CD & PS,O,CD & PS,P,CD & $G D, P, 3 \times 3$ & $\mathrm{CD}, \mathrm{P}$ & $\mathrm{CD}, \mathrm{NR}$ & FSCD \\
\hline iters for convergence & 18 & 12 & 11 & 14 & 11 & 11 & 11 \\
\hline CPU s for convergence & 23.3 & 17.4 & 15.1 & 18.1 & 44.3 & 52.3 & 56.2 \\
\hline CPU s per iteration & 1.2 & 1.3 & 1.2 & 1.1 & 3.8 & 4.6 & 4.9 \\
\hline $\begin{array}{l}\text { exponentiations } \\
\text { per iteration }\end{array}$ & 0 & 0 & 0 & 0 & M & $\mathrm{M}$ & $2 \mathrm{M}$ \\
\hline $\begin{array}{l}\text { add/subls } \\
\text { peritcration }\end{array}$ & $2 \mathrm{M}$ & $3 \mathrm{M}$ & $2 \mathrm{M}$ & $2 \mathrm{M}$ & $4 \mathrm{M}$ & $6 \mathrm{M}$ & $7 \mathrm{M}$ \\
\hline $\begin{array}{l}\text { mult/divs } \\
\text { per iteration }\end{array}$ & $3 \mathrm{M}$ & $5 \mathrm{M}$ & $3 \mathrm{M}$ & $2 \mathrm{M}$ & $5 \mathrm{M}$ & $11 \mathrm{M}$ & $10 \mathrm{M}$ \\
\hline $\begin{array}{l}\text { nonsequential accesses } \\
\text { per backprojection }\end{array}$ & M & $2 \mathrm{M}$ & M & M & $4 \mathrm{M}$ & $4 \mathrm{M}$ & $4 \mathrm{M}$ \\
\hline $\begin{array}{l}\text { nonsequential aceesses } \\
\text { per forward projection }\end{array}$ & $2 \mathrm{M}$ & $2 \mathrm{M}$ & $2 \mathrm{M}$ & M & M & M & M \\
\hline $\begin{array}{l}\text { system matrix accesses } \\
\text { per iteration }\end{array}$ & $2 \mathrm{M}$ & $2 \mathrm{M}$ & $2 \mathrm{M}$ & $2 \mathrm{M}$ & $2 \mathrm{M}$ & $2 \mathrm{M}$ & $2 \mathrm{M}$ \\
\hline
\end{tabular}

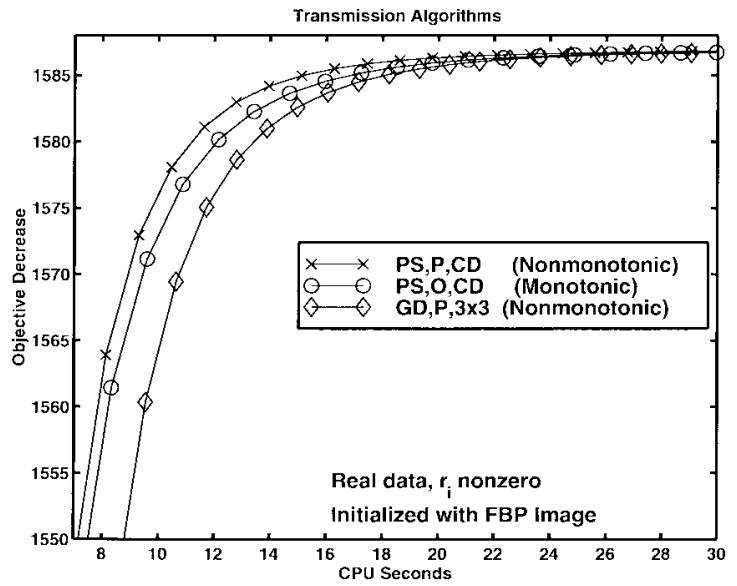

Fig. 7. Comparison of the speed of the proposed PS algorithms with the fastest algorithm that was introduced before: grouped CD with $3 \times 3$ groups.

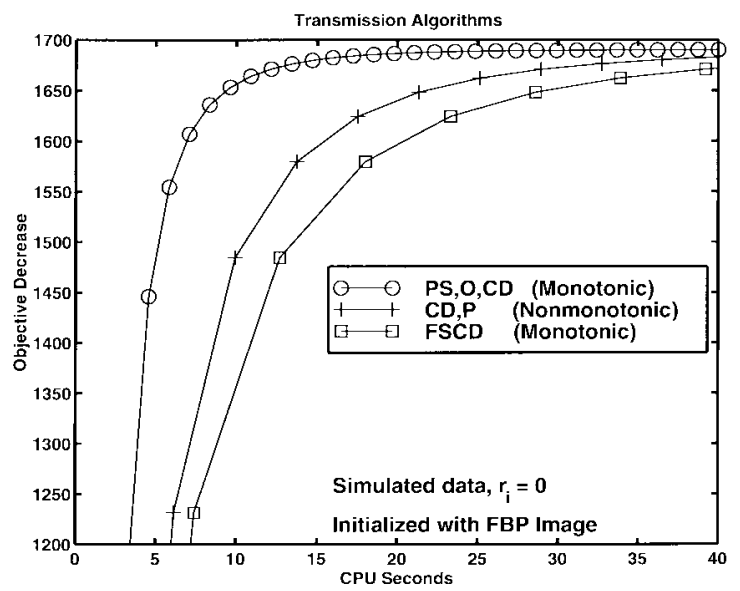

Fig. 8. Comparison of objective function decrease $\Phi\left(\mu^{0}\right)-\Phi\left(\mu^{n}\right)$ versus CPU time of monotonic PS and FS methods with CD. Note $r_{i}=0$ in this simulation.

ory accesses (of order $M$ only) per iteration are also tabulated, where $M$ is the number of nonzero entries in system matrix $\boldsymbol{A}$. For comparison purposes, a single forward and backprojection requires about $0.78 \mathrm{CPU}$ s. The $\mathrm{CD}$ and FS methods are significantly different from our proposed PSCD methods in the following respect. In our methods, the $\dot{q}_{i}$ terms are kept updated for all $i$ outside the projection loop in (38). In contrast, both CD and FS require $\dot{h}_{i}$ terms within the backprojection loop and these change with every pixel update so they must be computed on the fly within the backprojection loop. Thus, that backprojection must access $y_{i}, b_{i}, r_{i}, \hat{l}_{i}$ and the system matrix within the loop and perform quite a few floating point operations (including the exponentiations) with them. Not only is there inherently more floating point operations required for $\mathrm{CD}$ and FS, we suspect that the need to nonsequentially access parts of four sinogram-sized arrays, in addition to the system matrix, significantly degrades the ability of the CPU to pipeline operations. This leads to the dramatic differences in the CPU time between PSCD and CD methods.

If a monotonic algorithm is required, the PSCD algorithm with the optimal curvature (PS,O,CD) is the fastest algorithm. The other algorithms are not guaranteed to be monotonic except PSCD with maximum curvature. Although the PS,M,CD algorithm consumes less CPU time per iteration, it takes longer to converge since the curvatures result in an unnecessarily narrow surrogate function which causes small step sizes.

Among the nonmonotonic algorithms, another PS method, PSCD with precomputed curvatures (PS,P,CD) is the fastest. It converged in about $15 \mathrm{~s}$ with the real data used. The CPU time per iteration is the same as PS,M,CD, since they both precompute the denominator $\left(d_{j}^{n}\right)$ terms. Since the curvatures are smaller, this method decreases the objective very rapidly. Nevertheless, it is not guaranteed to be monotonic. However, as with the CD and GD with precomputed denominators [12], we have never observed any nonmonotonicity in practical applications with iterations started with an FBP image. The FSCD and CD algorithms consume a lot of CPU cycles per iteration and they are much slower than the proposed algorithms. The GD,P algorithm lowers the CPU requirements by decreasing the number of exponentiations, but it does not decrease the objective function as much per iteration as CD. Thus, it is also slightly slower than the PS,P,CD algorithm. This table shows that PSCD algorithms are preferable for both monotonic and nonmonotonic transmission image reconstructions. 


\section{CONCLUSION}

We have introduced a new class of algorithms for minimizing penalized-likelihood objective functions for transmission tomography. The algorithms are shown to be monotonic, even with the nonconvex objective function. In the nonconvex case there is no proof that these algorithms will find the global minimum, but at least the algorithms will monotonically decrease the objective function toward a local minimum. Practical experience suggests there are rarely multiple minima in this problem, but there is no proof. In the strictly convex case, the proposed algorithms are guaranteed to converge to the global minimum by a proof similar to that in [32].

Convergence is very important for algorithms for any optimization problem, particularly in medical applications. The PSCD algorithm is globally convergent when there are no background counts. Even when there are background counts, the new algorithm is guaranteed to monotonically decrease the objective function making the algorithm stable. Previous algorithms could not guarantee that property without expensive line searches. The robustness, stability, and speed of the new algorithm renders it usable in routine clinical studies. Such use should increase the emission image quality as compared to conventional methods which use linear processing and FBP for reconstruction. Further acceleration is possible by ordered subsets [33], albeit without guaranteed monotonicity.

The algorithms we introduced are simple, easy to understand, and fast. The simplicity, in part, is due to the additive form of (2), which is a direct consequence of independent measurements. Since the emission tomography log likelihood has a very similar form, due to independence of measurements, it is possible to apply the paraboloidal surrogates idea to the emission case, as well, to get faster algorithms [34].

It is possible to parallelize the PS algorithms by applying either the grouped descent (GD) [12], [13] algorithm to the surrogate function, or by parallelizing the projection and backprojection operators [35] for each pixel. However, in a serial computer we found that the PS method with GD update (PSGD) was not faster than the PSCD algorithm. This is due to the fact that the gradient updates in the PSCD algorithm consume much less CPU time than the gradient evaluations in the original CD algorithm, which require expensive exponentiations and floating point operations. Hence, grouped descent did not reduce the CPU time per iteration as much in the PS method as in the direct method.

In our opinion, the PS,O,CD algorithm supersedes all of our previous methods [4], [12], [18], and is our recommended algorithm for penalized-likelihood transmission tomography. The PS,P,CD algorithm is a faster but nonmonotonic alternative, which can be used for noncritical applications. A possible compromise would be to run a few iterations of the PS, O,CD algorithm and then fix the curvatures and denominator terms $\left(d_{j}^{m}\right)$ for the rest of the iterations to save computation time. Alternatively, one can run the PS,P,CD algorithm and check the objective function $\Phi(\mu)$ after each iteration to verify that it has decreased. If the objective does not decrease (which happens very rarely), then the PS,O,CD algorithm can be applied to the previous iterate to ensure monotonicity. For medical purposes, we believe that a monotonic algorithm should be used to reduce the risk of diagnostic errors due to erroneous reconstructions. Fortunately, with the new proposed methods, monotonicity can be assured with only a minor increase in CPU time (17.2 versus 15.1 CPU s).

\section{APPENDIX A}

We prove in this Appendix that the maximum second derivative of $h_{i}(l)$ for $l \geq 0$ is given by (14). We drop the subscript $i$ for simplicity.

The form of the $h$ functions is critical in the following. The second and third derivatives of the function $h$ in (3) are

$$
\begin{aligned}
\ddot{h}(l) & =\left(1-\frac{y r}{\left(b e^{-l}+r\right)^{2}}\right) b e^{-l} \\
h^{(3)}(l) & =\left(y r\left[\frac{-b e^{-l}+r}{\left(b e^{-l}+r\right)^{3}}\right]-1\right) b e^{-l} .
\end{aligned}
$$

We assume $b>0, y \geq 0$, and $r \geq 0$ throughout these appendices. First, we prove two lemmas about properties of these $h$ functions. These lemmas are used for the proofs in Appendix B as well.

Lemma 1: The following are equivalent for $h(l)$ defined in (3);

(E1) $r=0$ or $r \geq y$;

(E2) $h$ is strictly convex;

(E3) $\dot{h}$ is strictly concave;

(E4) $\dot{h}$ is monotonically increasing;

(E5) $\ddot{h}$ is monotonically decreasing.

Proof: Since $h$ is three times continuously differentiable, $h$ is strictly convex if and only if $\ddot{h}>0$ and $\dot{h}$ is strictly concave if and only if $h^{(3)}<0$. Clearly, $\ddot{h}>0$ if and only if $\dot{h}$ is monotonically increasing. So, (E2) $\Longleftrightarrow$ (E4). For similar reasons, $(\mathrm{E} 3) \Longleftrightarrow$ (E5).

If $r=0$ or $r \geq y$, then $y r<\left(b e^{-l}+r\right)^{2}$ so, from (30), $\ddot{h}(l) \geq 0, \forall l$. Thus, (E1) $\Rightarrow(\mathrm{E} 2)$.

To prove $(\mathrm{E} 1) \Rightarrow(\mathrm{E} 3)$, from (31), it suffices to show that $\left(b e^{-l}+r\right)^{3}>y r\left(-b e^{-l}+r\right)$. But this is trivial since $r^{3} \geq y r^{2}$ under the conditions (E1).

To prove the opposite, if $r \neq 0$ and $y>r$, then one can easily show that $\ddot{h}(l)$ and $-h^{(3)}(l)$ can take negative values for sufficiently large $l$ considering (30) and (31). So, (E2) $\Rightarrow$ (E1) and $(\mathrm{E} 3) \Rightarrow(\mathrm{E} 1)$.

Lemma 2: When $y>r$ and $r \neq 0$, the nonconvex function $\dot{h}$ has the following properties:

(P1) $\dot{h}$ is continuously differentiable;

(P2) $\dot{h}$ has exactly one critical point $l^{*}$, i.e., $\ddot{h}\left(l^{*}\right)=0$, and $l^{*}$ is a local maximizer of $\dot{h}(l)$;

(P3) $\dot{h}$ is strictly concave and monotone increasing for $l<l^{*}$

(P4) $\dot{h}$ is monotone decreasing for $l>l^{*}$;

(P5) $\ddot{h}$ has exactly one critical point $l^{z}$, i.e., $h^{(3)}\left(l^{z}\right)=0$ and $l^{z}$ is a local minimizer of $\ddot{h}(l)$.

Proof: (P1) is obvious from (21) and (30).

In the nonconvex case, the equation $\ddot{h}(l)=0$ has exactly one solution in $\mathbb{R}, l^{*}=\log (b /(\sqrt{y r}-r))$. Since $h^{(3)}\left(l^{*}\right)=$ $-2\left((\sqrt{y r}-r)^{2} / \sqrt{y r}\right)<0, l^{*}$ is a local maximum, proving (P2). 
Solutions to the equation $h^{(3)}(l)=0$ are the roots of a cubic polynomial in the variable $t=b e^{-l}$ which has only one real solution. The real root is negative when $h$ is convex, resulting in no solution for $l$. But, in the nonconvex case the real root is positive and results in exactly one solution $l^{z}=\log (b /(a / 3-y r / a-r))$ where

$$
a=\sqrt[3]{27 y r^{2}+3 \sqrt{3 y^{3} r^{3}+81 y^{2} r^{4}}} .
$$

So, $\ddot{h}(l)$ has exactly one critical point. We have shown above that $h^{(3)}\left(l^{*}\right)<0$ and one can easily see that $h^{(3)}(l) \approx$ $(y / r-1) b e^{-l}>0$ for large $l$. Thus, $l^{z}>l^{*}$ and $h^{(3)}(l)<0$ for $l<l^{z}$. So, $\ddot{h}(l)$ is monotonically decreasing for $l<l^{z}$. Also for $l>l^{z}, h^{(3)}(l)>0$, and $\ddot{h}(l)$ is monotonically increasing. This proves that $l^{z}$ is a local minimum for $\ddot{h}(l)$. Hence, (P5) is proven.

To prove (P3) we have to show $h^{(3)}(l)<0$ and $\ddot{h}(l)>0$ for $l<l^{*}$. However, as we found above $l^{*}<l^{z}$ and $h^{(3)}(l)<0$ for $l<l^{z}$. Also, $\ddot{h}(l)>0$ for $l<l^{*}$ since $l^{*}$ is the only critical point and local maximizer of $\dot{h}$ due to (P2). So, (P3) is also proven.

The function $\ddot{h}(l)$ has exactly one zero crossing $l^{*}$ from (P2) which is a local maximizer of $\dot{h}$. Then, $\ddot{h}(l)$ has to be always negative for $l>l^{*}$ proving (P4). To verify, one can easily see that $\ddot{h}(l) \approx(1-y / r) b e^{-l}<0$ for large $l$ values. So $\ddot{h}(l)<0 \forall l>l^{*}$.

The following result follows from (E5) of Lemma 1 for the convex case and from (P5) of Lemma 2 for the nonconvex case.

Corollary 1: The maximum value for $\ddot{h}$ in the region $[0, \infty)$ is achieved at the end points, i.e.,

$$
\begin{aligned}
c_{i}\left(l_{i}^{n}\right) & =\max _{l \in[0, \infty)}\{\ddot{h}(l)\}=\max \{\ddot{h}(\infty), \ddot{h}(0)\} \\
& =[\ddot{h}(0)]_{+}=\left[\left(1-\frac{y r}{(b+r)^{2}}\right) b\right]_{+} .
\end{aligned}
$$

The result follows since $\lim _{l \rightarrow \infty} \ddot{h}(l)=0$.

\section{APPENDIX B}

In this Appendix, we prove that the curvature defined in (28) is the optimum curvature that satisfies (27), which in turn implies from (26) that the choice (28) yields the fastest convergence rate. We first prove two lemmas about strictly concave functions.

Lemma 3: A 1-D line $l(x)=a x+b$ can intersect a strictly concave (or strictly convex) function $f(x)$ at most twice.

Proof: Suppose $l\left(x_{i}\right)=f\left(x_{i}\right)$ at points $x_{1}<x_{2}<$ $x_{3}$. Then since $f(x)$ is strictly concave, $f(x)>l(x)$ for $x \in\left(x_{1}, x_{3}\right)$, which contradicts the initial assumption that $f\left(x_{2}\right)=l\left(x_{2}\right)$.

Lemma 4: Let $f(x)$ be a 1-D strictly concave function, and let $l(x)=a x+b$ be a line that intersects $f(x)$ at the two points $x_{1}<x_{2}$. Then

$$
f(x)<l(x) \text { for } x \in\left(-\infty, x_{1}\right) \cup\left(x_{2}, \infty\right) .
$$

Proof: Suppose there exists an $x_{3}>x_{2}$ such that $f\left(x_{3}\right) \geq l\left(x_{3}\right)$. Consider the new line $m(x)$ that intersects $f(x)$ at $x_{1}$ and $x_{3}$. Since $m\left(x_{1}\right)=l\left(x_{1}\right)$ and $m\left(x_{3}\right)=$ $f\left(x_{3}\right) \geq l\left(x_{3}\right)$, it follows from the affine form of $l(x)$ and $m(x)$ that $m\left(x_{2}\right) \geq l\left(x_{2}\right)=f\left(x_{2}\right)$, which contradicts the assumption that $f(x)$ is strictly concave. The case $x_{3}<x_{1}$ is similar.

For simplicity in this appendix, we drop the subscript $i$ and the dependence on $n$ for the variables. Let $h(l)$ be the marginal negative log-likelihood function defined in (3) with derivatives presented in (21), (30), and (31) and let $q(l)$ be the parabolic surrogate function defined in (8) with the optimum curvature $c$ defined in (28). We use $l^{c}$ to denote the current projection value $l_{i}^{n}$. The reader may visualize the following proofs by considering the plots of $\dot{h}$ and $\dot{q}$ functions shown in Fig. 3 .

We define the difference function by

$$
\delta(l) \triangleq q(l)-h(l) .
$$

To show that $q(l) \geq h(l)$ for $l \geq 0$, as required by (9), it suffices to show that $\delta(l) \geq 0$. When $l^{c}=0$, it is obvious from Appendix A that $\delta(l) \geq 0$. Thus, we focus on the case $l^{c}>0$ in the following.

Lemma 5: The following conditions are sufficient to ensure $\delta(l) \geq 0, \forall l \in[0, \infty)$.

(C1) $\delta(0) \geq 0$ and $\delta\left(l^{c}\right)=0$;

(C2) $\dot{\delta}(l) \geq 0$ for $l \geq l^{c}$; and

(C3) either

(C31) $\dot{\delta}(l)<0, \forall l \in\left[0, l^{c}\right)$, or

(C32) $\exists l^{p} \in\left[0, l^{c}\right)$ such that $\dot{\delta}(l) \geq 0$ for $l \in\left[0, l^{p}\right]$ and $\dot{\delta}(l) \leq 0$ for $l \in\left(l^{p}, l^{c}\right]$.

Proof: Since $\delta\left(l^{c}\right)=0$

$$
\delta(l)=\int_{l^{c}}^{l} \dot{\delta}(t) d t
$$

Case $l \geq l^{c}$ : The integrand in (33) is nonnegative due to (C2), so $\delta(l) \geq 0$.

Case $l \in\left[0, l^{c}\right]:$ If $(\mathrm{C} 31)$ is true, then $\delta(l)=\delta\left(l^{c}\right)-$ $\int_{l}^{l^{c}} \dot{\delta}(t) d t \geq \delta\left(l^{c}\right)=0$.

If (C32) holds and $l \in\left[0, l^{p}\right]$, then $\delta(l)=\delta(0)+$ $\int_{0}^{l} \dot{\delta}(t) d t \geq \delta(0) \geq 0$ by (C1). Likewise, if (C32) holds and $l \in\left(l^{p}, l^{c}\right]$, then $\delta(l)=\delta\left(l^{c}\right)-\int_{l}^{l^{c}} \dot{\delta}(t) d t \geq \delta\left(l^{c}\right)=0$ again by $(\mathrm{C} 1)$.

Hence, $\delta(l) \geq 0 \forall l \geq 0$ under the above conditions.

We now establish the conditions of Lemma 5. (C1) follows directly from the definition (27), so we focus on (C2) and (C3) below. We first treat the case where $h(l)$ is strictly convex.

Lemma 6: If $h(l)$ is a convex function and $\dot{h}(l)$ is concave for $l \geq 0$, then the difference function $\delta(l)$ in (32) with the curvature $c$ defined in (28) satisfies conditions (C2) and (C32) in Lemma 5. Furthermore, $c>0$.

Proof: It is trivial to show that the conditions (E2) through (E5) of Lemma 1 hold in this case for $l \geq 0$. First we prove $c>0$. Suppose $c=0$, so $\dot{q}$ is a constant. Since $\dot{h}(l)$ is increasing by (E4) in Lemma 1 and $\dot{q}\left(l^{c}\right)=\dot{h}\left(l^{c}\right)$, it is obvious that $\dot{q}(l)>\dot{h}(l), \forall l \in\left[0, l^{c}\right)$, so $\delta(0)=-\int_{0}^{l^{c}} \dot{\delta}(t) d t<0$ contradicting (C1). So, $c>0$ in this case and $\delta(0)=0$ by design. 
To prove (C32), consider $\dot{h}$. The line $\dot{q}$ cannot intersect the strictly concave $h$ at more than two points due to Lemma 3 . We know that $\dot{\delta}\left(l^{c}\right)=0$, thus $l^{c}$ is an intersection point. We have $\delta(0)=0$ and $\delta\left(l^{c}\right)=0$ by definition. From mean value theorem, there must be another intersection point $l^{p} \in\left[0, l^{c}\right)$ such that $\dot{\delta}\left(l^{p}\right)=0$. We know by Lemma 3 that there cannot be any additional points where $\dot{\delta}(l)=0 . \dot{\delta}(l)<0$ for $l \in\left(l^{p}, l^{c}\right)$ due to concavity of $\dot{h}$ and $\dot{\delta}(l)>0$ for $l \in\left[0, l^{p}\right)$ due to Lemma 4. (C32) is proven.

To prove (C2), apply Lemma 4 to the strictly concave function $\dot{h}$ with two points $l^{p}$ and $l^{c}$ as the intersection points of the line with the curve.

We now consider the realistic nonconvex case.

Lemma 7: Let $h(l)$ be a nonconvex function with its derivative $\dot{h}$ satisfying properties (P1), (P2), and (P3) in Lemma 2. The difference function $\delta(l)$ defined in (32) with the curvature defined in (28) satisfies (C2) and (C3) in Lemma 5.

Proof: The reader can refer to Fig. 3 for representative plots of $h$ and its first derivative. Note that in Lemma 2, (P2) $\Rightarrow$ (P4) directly.

Consider these two cases where $l^{*}$ is defined as in Lemma 2:

Case $l^{c}<l^{*}$ : By (P3) of Lemma 2, $l^{c}$ is in a concave increasing region. By Lemma 6, (C32) holds as well as the fact that $c>0$. To prove (C2), we use property (P4), that $\dot{h}$ is a decreasing function for $l>l^{*}$. So, since $\dot{q}\left(l^{*}\right)>\dot{h}\left(l^{*}\right)$ [as for (C2) in Lemma 6 again] and $c \geq 0, \dot{q}(l)>\grave{h}(l), \forall l \geq l^{c}$.

Case $l^{c} \geq l^{*}$ : Since by $(\mathrm{C} 1), \delta\left(l^{c}\right)-\delta(0)=\int_{0}^{l^{c}} \dot{\delta}(t) d t \leq$ $0, \dot{\delta}(l)=\dot{q}(l)-\dot{h}(l)$ cannot always be nonnegative over the interval $\left[0, l^{c}\right)$. So, either $\dot{q}(l)<\dot{h}(l), \forall l \in\left[0, l^{c}\right)$ or $\dot{q}$ intersects $\dot{h}(\dot{\delta}(l)=0)$ at least once in $\left[0, l^{c}\right)$. If the former case occurs, (C31) holds by definition. If the latter case occurs, then we have to prove that (C32) holds, i.e., there is no more than one point at which $\dot{q}$ intersects $\dot{h}$ in $\left[0, l^{c}\right)$. Since $c \geq 0$ and $\dot{h}$ is decreasing in the region $l>l^{*}$, the intersection point(s) $l^{p}<l^{*}$. We cannot apply Lemma 3 here to prove that there is no other intersection point, but we can use Lemma 4 to prove it. Assume there is another intersection point. Then, the function $\dot{q}>\dot{h}$ in the concave region outside the interval between two intersection points by Lemma 4 which implies $\dot{\delta}\left(l^{*}\right)>0$ and $\dot{\delta}(l)>0$ for $l>l^{*}$. But this would contradict the fact that $\dot{\delta}\left(l^{c}\right)=0$. So, (C32) must hold.

In this case, the fact that $c \geq 0$ is enough to prove (C2), since $\dot{h}$ is decreasing in this region.

Theorem 1: Let $h(l)$ be a 1-D function that satisfies either of the following:

(H1) $h(l)$ is strictly convex and $\dot{h}(l)$ is strictly concave in the feasible region $l \geq 0$ or

(H2) $\dot{h}(l)$ satisfies (P1), (P2), and (P3) of Lemma 2.

Then the curvature defined in (28) satisfies the optimality condition in (27).

Proof: For $h$ functions that satisfy conditions (H1), Lemma 6 with Lemma 5 prove that the curvature (28) satisfies (9) for $l^{c}>0$. For $h$ functions satisfying conditions in (H2), Lemmas 7 and 5 similarly prove that the curvature (28) satisfies (9) for $l^{c}>0$. The rest of the proof applies to both cases (H1) and (H2). For $l^{c}=0, c$ in (28) is the maximum second derivative in $[0, \infty)$, and (9) is satisfied by mean value theorem as mentioned in Section III-A.

We need to prove that no other nonnegative curvature less than (28) satisfies (9).

Assume $0 \leq c^{*}<c$, and let

$$
q^{*}(l)=h\left(l^{c}\right)+\dot{h}\left(l^{c}\right)\left(l-l^{c}\right)+\frac{1}{2} c^{*}\left(l-l^{c}\right)^{2} .
$$

Obviously $c^{*}$ can exist only when $c>0$ since $c=0$ is the minimum curvature we allow. With $c>0$, it is obvious from (28) that $q(0)=h(0)$. If $l^{c}>0$, this clearly implies that $q^{*}(0)<q(0)=h(0)$ which shows that $c^{*}$ cannot satisfy (9). If $l^{c}=0$, then a curvature $c^{*}<c$ would force $\dot{q}$ to lie under $\dot{h}$ for some small values of $l$. That is, $\exists \epsilon>0$ such that $q(l)<h(l)$ for $\epsilon>l>0$. Thus $c^{*}$ does not satisfy (9) even for $l^{c}=0$.

Corollary 2: The "optimum curvature" defined in (28) using the marginal negative log-likelihood function $h_{i}(l)$ defined in (3) for the transmission tomography problem satisfies the optimality condition in (27) for $b_{i}>0, y_{i} \geq 0, r_{i} \geq 0$.

Proof: The function $h_{i}(l)$ defined in (3) satisfies the conditions $(\mathrm{H} 1)$ or $(\mathrm{H} 2)$ of Theorem 1 depending on the values of $y_{i}$ and $r_{i}$ as shown in Lemmas 1 and 2. Hence Theorem 1 is directly applicable to the transmission tomography problem.

\section{ACKNOWLEDGMENT}

The authors gratefully acknowledge discussions with G. Gualtieri and K. Lange, and would also like to thank J. Webster Stayman for implementing LBFGS.

\section{REFERENCES}

[1] S. C. Huang, E. J. Hoffman, M. E. Phelps, and D. E. Kuhl, "Quantitation in positron emission computed tomography: 2 effects of inaccurate attenuation correction," J. Computer-Assisted Tomogr., vol. 3, no. 6, pp. 804-814, Dec. 1979 .

[2] H. Erdoğan and J. A. Fessler, "Statistical image reconstruction methods for simultaneous emission/transmission PET scans," in Proc. IEEE Nuclear Science Symp. Medical Imaging Conf., 1996, vol. 3, pp. 1579-1583.

[3] E. U. Mumcuoglu, R. Leahy, S. R. Cherry, and Z. Zhou, "Fast gradientbased methods for Bayesian reconstruction of transmission and emission PET images," IEEE Trans. Med. Image., vol. 13, pp. 687-701, Dec. 1994.

[4] J. A. Fessler, "Hybrid Poisson/polynomial objective functions for tomographic image reconstruction from transmission scans," IEEE Trans. Image Processing, vol. 4, no. 10, pp. 1439-1450, Oct. 1995.

[5] A. P. Dempster, N. M. Laird, and D. B. Rubin, "Maximum likelihood from incomplete data via the EM algorithm," J. R. Stat. Soc. Ser. B, vol. 39 , no. 1 , pp. $1-38,1977$

[6] K. Lange and R. Carson, "EM reconstruction algorithms for emission and transmission tomography," J. Computer-Assisted Tomogr., vol. 8, no. 2, pp. 306-316, Apr. 1984.

[7] J. M. Ollinger, "Maximum likelihood reconstruction of transmission images in emission computed tomography via the EM algorithm," IEEE Trans. Med. Image., vol. 13, pp. 89-101, Mar. 1994.

[8] J. T. Kent and C. Wright, "Some suggestions for transmission tomography based on the EM algorithm," in Stochastic Models, Statistical Methods, and Algorithms in Image Analysis (Lecture Notes in Statistics) M. Piccioni, P. Barone, and A. Frigessi, Eds. New York: SpringerVerlag, 1992, vol. 74, pp. 219-232.

[9] J. A. Browne and T. J. Holmes, "Developments with maximum likelihood X-ray computed tomography," IEEE Trans. Med. Image., vol. 12, pp. 40-52, Mar. 1992.

[10] C. A. Bouman and K. Sauer, "A unified approach to statistical tomography using coordinate descent optimization," IEEE Trans. Image Processing, vol. 5, no. 3, pp. 480-492, Mar. 1996. 
[11] K. Sauer and C. Bouman, "A local update strategy for iterative reconstruction from projections," IEEE Trans. Signal Processing, vol. 41, no. 2, pp. 534-548, Feb. 1993.

[12] J. A. Fessler, E. P. Ficaro, N. H. Clinthorne, and K. Lange, "Groupedcoordinate ascent algorithms for penalized-likelihood transmission image reconstruction," IEEE Trans. Med. Image., vol. 16, pp. 166-175, Apr. 1997.

[13] K. D. Sauer, S. Borman, and C. A. Bouman, "Parallel computation of sequential pixel updates in statistical tomographic reconstruction," in Proc. IEEE Int. Conf. Image Processing, 1995, vol. 3, pp. 93-96.

[14] A. R. De Pierro, "On the relation between the ISRA and the EM algorithm for positron emission tomography," IEEE Trans. Med. Image., vol. 12, pp. 328-333, June 1993.

[15] J. Zheng, S. Saquib, K. Sauer, and C. Bouman, "Functional substitution methods in optimization for Bayesian tomography," IEEE Trans. Image Processing, Mar. 1997, submitted for publication.

[16] S. Saquib, J. Zheng, C. A. Bouman, and K. D. Sauer, "Provably convergent coordinate descent in statistical tomographic reconstruction,' in Proc. IEEE Intl. Conf. Image Processing, 1996, vol. 2, pp. 741-744.

[17] A. R. De Pierro, "A modified expectation maximization algorithm for penalized likelihood estimation in emission tomography," IEEE Trans. Med. Image., vol. 14, pp. 132-137, Mar. 1995.

[18] K. Lange and J. A. Fessler, "Globally convergent algorithms for maximum a posteriori transmission tomography," IEEE Trans. Image Processing, vol. 4, pp. 1430-1438, Oct. 1995

[19] P. J. Huber, Robust Statistics. New York: Wiley, 1981.

[20] E. Ü. Mumcuoğlu, R. M. Leahy, and S. R. Cherry, "Bayesian reconstruction of PET images: Methodology and performance analysis," Phys. Med. Biol., vol. 41, no. 9, pp. 1777-1807, Sept. 1996.

[21] M. Yavuz and J. A. Fessler, "New statistical models for randomsprecorrected PET scans," in Information Processing in Medical Imaging (Lecture Notes in Computer Science), J. Duncan and G. Gindi, Eds. Berlin, Germany: Springer-Verlag, 1997, vol. 1230, pp. 190-203.

[22] S. R. Meikle, M. Dahlbom, S. R. Cherry, and A. Chatziioannou, "Attenuation correction in whole body PET," J. Nucl. Med. (Abstract Book), vol. 33, no. 5, p. 862, May 1992.

[23] J. A. Fessler, "Grouped coordinate descent algorithms for robust edgepreserving image restoration," in Proc. SPIE 3071, Image Reconstruction Restoration II, 1997, pp. 184-194.
[24] P. G. Ciarlet, Introduction to Numerical Linear Algebra and Optimization. Cambridge, U.K. : Cambridge Univ. Press, 1982.

[25] J. A. Fessler and A. O. Hero, "Penalized maximum-likelihood image reconstruction using space-alternating generalized EM algorithms," IEEE Trans. Image Processing, vol. 4, no. 10, pp. 1417-1429, Oct. 1995.

[26] J. A. Fessler, N. H. Clinthorne, and W. L. Rogers, "On complete data spaces for PET reconstruction algorithms," IEEE Trans. Nucl. Sci., vol. 40, pp. 1055-1061, Aug. 1993.

[27] J. A. Fessler, "Penalized weighted least-squares image reconstruction for positron emission tomography," IEEE Trans. Med. Image., vol. 13, pp. 290-300, June 1994.

[28] K. Wienhard, L. Eriksson, S. Grootoonk, M. Casey, U. Pietrzyk, and W. D. Heiss, "Performance evaluation of a new generation positron scanner ECAT EXACT," J. Computer-Assisted Tomogr., vol. 16, no. 5, pp. 804-813, Sept. 1992.

[29] J. A. Fessler and W. L. Rogers, "Spatial resolution properties of penalized-likelihood image reconstruction methods: Space-invariant tomographs," IEEE Trans. Image Processing, vol. 5, pp. 1346-1358, Sept. 1996

[30] K. Lange, "Convergence of EM image reconstruction algorithms with Gibbs smoothing," IEEE Trans. Med. Image., vol. 9, pp. 439-446, Dec. 1990. (See also Errata in vol. 10, pp. 288, June 1991.

[31] C. Zhu, R. H. Byrd, P. Lu, and J. Nocedal, "Algorithm 778: L-BFGSB: Fortran subroutines for large-scale bound-constrained optimization," ACM Trans. Math. Software, vol. 23, no. 4, pp. 550-560, Dec. 1997.

[32] J. A. Fessler and A. O. Hero, "Space-alternating generalized expectationmaximization algorithm," IEEE Trans. Signal Processing, vol. 42, pp. 2664-2677, Oct. 1994.

[33] H. Erdoğan, G. Gualtieri, and J. A. Fessler, "An ordered subsets algorithm for transmission tomography," in Proc. IEEE Nuclear Science Symp. Medical Imaging Conf., 1998. (Inadvertently omitted from Proceedings.) Available: http://www.eecs.umich.edu/fessler

[34] J. A. Fessler and H. Erdoğan, "A paraboloidal surrogates algorithm for convergent penalized-likelihood emission image reconstruction," in Proc. IEEE Nuclear Science Symp. Medical Imaging Conf., 1998, vol. 2, pp. 1132-1135.

[35] W. Niethammer, "A note on the implementation of the successive overrelaxation method for linear complementarity problems," Numerical Algorithms, vol. 4, no. 1, pp. 197-200, Jan. 1993. 